\title{
Tezacaftor and ivacaftor for the treatment of cystic fibrosis
}

DOI:

10.1080/17476348.2020.1682998

\section{Document Version}

Accepted author manuscript

Link to publication record in Manchester Research Explorer

\section{Citation for published version (APA):}

Paterson, S., Barry, P., \& Horsley, A. (2019). Tezacaftor and ivacaftor for the treatment of cystic fibrosis. Expert review of respiratory medicine. https://doi.org/10.1080/17476348.2020.1682998

\section{Published in:}

Expert review of respiratory medicine

\section{Citing this paper}

Please note that where the full-text provided on Manchester Research Explorer is the Author Accepted Manuscript or Proof version this may differ from the final Published version. If citing, it is advised that you check and use the publisher's definitive version.

\section{General rights}

Copyright and moral rights for the publications made accessible in the Research Explorer are retained by the authors and/or other copyright owners and it is a condition of accessing publications that users recognise and abide by the legal requirements associated with these rights.

\section{Takedown policy}

If you believe that this document breaches copyright please refer to the University of Manchester's Takedown Procedures [http://man.ac.uk/04Y6Bo] or contact uml.scholarlycommunications@manchester.ac.uk providing relevant details, so we can investigate your claim.

\section{OPEN ACCESS}




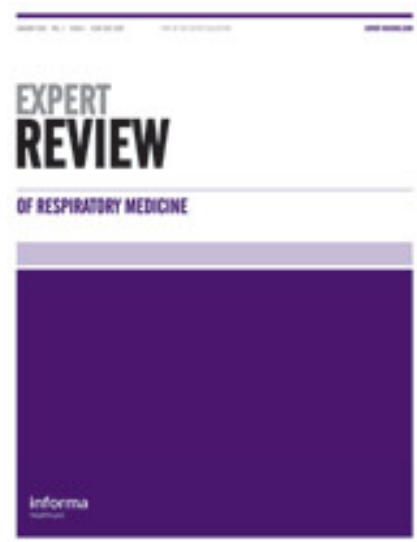

Tezacaftor and ivacaftor for the treatment of cystic fibrosis

\begin{tabular}{|r|l|}
\hline Journal: & Expert Review of Respiratory Medicine \\
\hline Manuscript ID & ERRX-2019-ST-0134.R1 \\
\hline Manuscript Type: & Drug profile (invited) \\
\hline Keywords: & Cystic fibrosis, CFTR, Ivacaftor, Lumacaftor, tezacaftor \\
\hline $\begin{array}{l}\text { Note: The following files were submitted by the author for peer review, but cannot be converted to PDF. } \\
\text { You must view these files (e.g. movies) online. }\end{array}$ \\
\hline Figure 1- Pictorial representation of distinct CFTR class mutations..pptx \\
\hline
\end{tabular}

\section{SCHOLARONE ${ }^{\mathrm{M}}$ Manuscripts}


Article type: Drug Profile

Tezacaftor and ivacaftor for the treatment of cystic fibrosis

Sarah L Paterson*1,2, Peter J Barry ${ }^{1,2}$, Alexander R Horsley ${ }^{1,2}$

${ }^{1}$ Manchester Adult Cystic Fibrosis Centre, Manchester NHS Foundation Trust, Wythenshawe Hospital, Southmoor Road, Wythenshawe, M23 9LT

${ }^{2}$ Division of Infection Immunity \& Respiratory Medicine, Faculty of Biology Medicine and Health, University of Manchester, Manchester, UK

*Corresponding author:

Sarah L Paterson

Manchester Adult Cystic Fibrosis Centre,

Manchester NHS Foundation Trust, Wythenshawe Hospital,

Southmoor Road, Wythenshawe, M23 9LT

Email: sarah.paterson@mft.nhs.uk 


\begin{abstract}
Introduction: Cystic fibrosis (CF) is a complex, multi-system, genetic disease affecting over 70,000 people worldwide. The underlying defect is a mutation in the CFTR gene.

Dysfunctional CFTR protein results in abnormal anion movement across epithelial membranes in affected organs. There has been a paradigm shift in CF treatment over the last decade with the advent of CFTR modulation, treatments which target this underlying genetic defect and have the potential to change the course of CF clinical disease.
\end{abstract}

Areas covered: Available CFTR modulators in current clinical practice are reviewed in this article, with a direct comparison and summary of relevant pivotal clinical trials. The approval of ivacaftor and subsequent development of lumacaftor and tezacaftor dual combinations represent an exciting development in CF management in recent years.

Expert opinion: Tezacaftor/ivacaftor (tez/iva) appears to have a more favourable adverse event and drug-drug interaction profile than lumacaftor/ivacaftor. Tez/iva has been approved, alongside Phe508del, for a large number of 'residual function' CFTR mutations, with some based on response in in vitro culture. Dual therapy with tez/iva has paved the way for triple CFTR modulation currently in clinical trials with an ultimate view to provide modulation therapy to the majority of CF genotypes in the future. 


\section{Article highlights}

- CFTR modulation is an ever-expanding field within the armamentarium of CF treatment, and has changed the landscape of CF management in the last decade.

- Ivacaftor was the first successful CFTR modulator developed and implemented into clinical practice, approved for Gly551Asp mutations.

- The development of lumacaftor/ivacaftor (lum/iva) demonstrated that patients homozygous for the Phe508del mutation could gain clinical benefit from CFTR modulators. However, issues with tolerability and some important drug-drug interactions are challenges which have encouraged the development of alternative compounds

- Tezacaftor/ivacaftor (tez/iva) is the most recent CFTR modulator to be approved for clinical use in the EU and US, for Phe508del homozygotes and Phe508del heterozygotes with and certain 'residual function' mutations (table 3).

- Clinical efficacy has been shown in tezacaftor/ivacaftor phase 3 trials, evidenced by improvements in absolute $\mathrm{ppFE}_{1}$, pulmonary exacerbation rates and respiratory symptom scores (CFQ-R). Reductions in sweat chloride have been shown, but to a lesser extent than ivacaftor monotherapy.

- Tez/iva appears to be better tolerated in terms of respiratory adverse events and has less drug-drug interactions (DDI's) compared to its predecessor lum/iva.

- Tez/iva has been taken forward into phase 3 trials of triple combination CFTR modulator therapy, alongside next generation CFTR correctors, showing promising preliminary results.

- Longitudinal patient data will be important to analyse in order to establish evidence of longer-term efficacy of CFTR modulation therapy, particularly dual and triple combinations.

- The development of triple combination CFTR modulators incorporating tezacaftor and ivacaftor will broaden the indications for therapy to the vast majority of the CF population. 


\subsection{Introduction}

Cystic fibrosis (CF) is a complex, autosomal recessive, multisystem disease, affecting approximately 1 in 2500-3500 live births in the UK(1). The condition is caused by mutations in a gene located on the long arm of chromosome 7 encoding a membrane protein known as the cystic fibrosis transmembrane conductance regulator (CFTR). The CFTR protein acts predominantly as a regulator of anion movement across exocrine gland epithelial cell membranes. CFTR is expressed throughout the body and affects secretory function in multiple organs including the lungs, pancreas, gastro-intestinal tract, liver, genitourinary system and sweat glands(2).

Mutations of the CFTR gene can result in abnormal ion movement across epithelial cell membranes. More than 2000 mutations have been identified in the gene(3), though substantially fewer have conclusively been found to result in defective anion channel function. If pathogenic, mutations result in reduction of chloride and bicarbonate at the cell surface. A lack of CFTR-mediated inhibition of epithelial sodium channels also causes excess movement of sodium ions into cells, enhancing osmotic water resorption. This leads to inadequate hydration of mucous secretions and defective mucociliary clearance. In the respiratory tract, retained secretions encourage airway blockage, bacterial adherence and a perpetuating cycle of infection, inflammation and tissue destruction(4).

Treatments for CF have expanded massively over recent decades, and life expectancy has increased substantially as a result. Traditional CF therapies have been aimed at mitigating the consequences of disease by improving airway clearance, reducing infective burden and 
supplementing absent pancreatic enzymes. New therapies directed towards improving CFTR function, either by increasing the amount of CFTR protein present at the cell surface (known as "CFTR correctors") or the activity of the channels that are present ("CFTR potentiators"), or in combination, are the first clinically available therapies directed at the root cause of CF. These represent a paradigm shift in the approach to CF treatment, and this area of CF therapeutics has progressed rapidly in the last decade. In this review, we will look at the evidence supporting the use of the combination therapy tezacaftor/ivacaftor (also known as Symkevi/Kalydeco ${ }^{\circledR}$ in the EU and Symdeko ${ }^{\circledR}$ in the US), consisting of a CFTR corrector and a potent CFTR potentiator. This therapy has received licensing in many territories globally for use in CF and forms the backbone of future "triple combination" therapies already in phase 3 trials.

\subsection{Intracellular processing of CFTR}

CFTR gene mutations have historically been classed into six groups according to the degree of protein malfunction. Although broad correlations between CFTR mutation class and disease severity exist, both environmental factors and modifier genes contribute to individual variation in clinical disease within CFTR mutation classes(5). Gene mutation classes however have more relevance in the context of potential response to CFTR modulating therapies.

Genetic classes of CFTR mutation are divided according to their effect on CFTR protein quantity and function. CFTR quantity is determined by correct synthesis and processing of CFTR before it reaches the cell membrane and its subsequent surface stability. CFTR function is determined by both the opening ability of the CFTR membrane channel and appropriate conductance of CFTR-driven anion movement across this channel. 
Class I and II CFTR mutations encompass defective synthesis and processing of CFTR protein. These tend to confer the most severe clinical disease, since there is little or no CFTR protein expressed at the cell surface. Class I CFTR mutations encompass frameshift or nonsense mutations, encoding premature terminations in mRNA sequence which results in no normal CFTR protein expression and severe clinical disease. Class II mutations result in defective CFTR protein maturation and trafficking to the epithelial cell surface. Class III CFTR abnormalities manifest as reduced anion channel opening at the cell surface and are also known as gating mutations. Class IV CFTR abnormalities affect chloride conductance across epithelial membranes via this anion channel. Whereas class III mutations are deemed to confer a severe phenotype, the variable conductance seen in class IV mutations has been associated with a milder clinical phenotype and better prognosis. Class V and VI CFTR mutations account for splicing abnormalities in DNA protein coding and result in less functional protein expression at the cell surface(6). A summary of CFTR mutation classes is shown in table 1. A pictorial representation of CFTR mutations at cellular level are shown in figure 1.

In practice, the distinction between genotype classes may be less defined and both genetic overlap and a varying spectrum of CFTR activity exist between groups(4).

The most common CFTR mutation in the UK CF population is the Phe508del mutation (formerly F508del), a class II mutation, accounting for one or both abnormal CFTR gene alleles in $89.5 \%$ of patients in the UK in $2017(8)$. This is also the commonest CFTR mutation worldwide, with approximately $45.3 \%$ of CF patients in the US homozygous for the Phe508del mutation(9). This mutation, a deletion of three base pairs encoding 
phenylalanine at residue 508, results in abnormal folding of Phe508del CFTR protein, causing instability and the potential for premature degradation(10). As a consequence, there is incorrect trafficking of CFTR protein to the apical membrane. This results in little to no normal CFTR activity and homozygous Phe508del CF patients typically have a severe clinical phenotype. Due to the high frequency of this abnormality, targeting the defective protein formed as a result of the Phe508del mutation has become a key target for the pharmaceutical industry.

\subsection{CFTR modulators in clinical practice}

There are currently three CFTR modulator preparations available for clinical use worldwide. These include ivacaftor, lumacaftor/ivacaftor (lum/iva) and most recently tezacaftor/ivacaftor (tez/iva). The mechanism of action of these novel therapies is to improve the activity of the dysfunctional protein formed as a result of genetic mutations, in order to reduce symptom burden and ideally prevent further downstream organ dysfunction. In this way, there is potential for these compounds to be truly diseasemodifying. Salient outcomes of the key CFTR modulator trials to date are summarised in table 2.

\subsection{Clinical efficacy, safety and tolerability of available CFTR modulators}

\subsection{Ivacaftor (VX-770, Kalydeco)}

Ivacaftor (Kalydeco ${ }^{\circledR}$ ) was the first CFTR modulator developed and approved for clinical use. Ivacaftor is a CFTR 'potentiator' and its mechanism of action is to increase the 'open probability' $\left(\mathrm{P}_{\mathrm{o}}\right)$ of the CFTR protein channel at the cell surface. As such, ivacaftor augments 
chloride ion transport across epithelial membranes when protein is correctly placed in the cell(11). Ivacaftor was originally licensed for treatment of CFTR gating mutations (class III) with abnormal CFTR channel opening, the commonest of which is Gly551Asp. This class represent around $5 \%$ of mutant alleles in CF patients worldwide(12). The proof of concept of ivacaftor efficacy was first shown in Gly551Asp/Phe508del human bronchial epithelial cells (HBE) in vitro, demonstrating an increase in CFTR channel $\mathrm{P}_{\mathrm{o}}$ and enhanced chloride secretion, leading to an improvement in cell surface liquid composition as well as correction of ciliary movement to mirror those of non-CF cells(13).

The phase 3 STRIVE(14) and ENVISION(15) trials revealed that ivacaftor produced a dramatic reduction in sweat chloride levels in CF patients aged six years and above. There were statistically and clinically significant improvements in lung function (measured by percentage of predicted forced expiratory volume in one second, $\mathrm{ppFEV}_{1}$ ) and body mass index (BMI) at 48 weeks, and a reduction in pulmonary exacerbations. An absolute ppFEV 1 improvement of over $10 \%$ was shown in the ivacaftor groups of both trials. The subsequent open label PERSIST study(16) showed this benefit to be sustained after 144 weeks of ivacaftor treatment with no new safety concerns. Importantly the efficacy demonstrated in clinical trials was also translated into similar effectiveness in patients with severe disease, a population excluded from the clinical trials(17).

Ivacaftor was subsequently shown to increase CFTR channel $P_{0}$ in a range of other gating mutations, including Gly178Arg, Ser549Asn, Ser549Asn, Gly551Ser, Gly970Arg, Gly1244Glu, Ser1251Asn, Ser1255Prp, Gly1349Asp, as well as wild-type (normal) CFTR(11). In addition, phase 3 trial data reported by Moss and colleagues in 2015 proved ivacaftor to be effective 
in adults with an Arg117His 'residual function' (RF) CFTR mutation, observing improvement in sweat chloride, CF-specific symptom scores and, although not significant, an increase in absolute $\mathrm{ppFEV}_{1}$ in this cohort(18).

The KIWI study(19) reported safety and tolerability data of ivacaftor in CF patients aged two to five years of age with a CFTR gating mutation. Reduction in sweat chloride levels of a similar magnitude to older ivacaftor patients were seen with weight-adjusted ivacaftor dosing, in addition to improvement in nutritional and exocrine pancreatic status.

\subsubsection{Adverse events}

Pooled data from phase 3 trials(14)(15) showed a satisfactory adverse event profile of ivacaftor, with only $2 \%$ drop-out or discontinuation rate. The most prevalent adverse events included headache, upper respiratory tract infection, nausea and nasal congestion. Ivacaftor has not been associated with significant pulmonary adverse events, however there is a risk of hepatotoxicity and liver function must be closely monitored following initiation of therapy. This is particularly important in CF patients with pre-existing liver disease, which may not preclude them from commencing CFTR modulation.

In the STRIVE(14) and ENVISION(15) trials, four patients (4\%) in the ivacaftor group had elevated ALT and AST levels $\geq 8 x$ upper limit of normal (ULN) within the 48-week period and nine patients had elevations between 5-8x ULN up to 144 weeks of treatment, all resulting in dose interruption but no discontinuation of therapy. In the KIWI study, significantly elevated liver transaminase levels were recorded to a higher degree than in previous ivacaftor studies but lacked a healthy control cohort for comparison(19). 
Current guidelines therefore require that liver function be monitored every three months in the first year of ivacaftor treatment and annually thereafter. Ivacaftor must be used with caution in CF patients with pre-existing liver disease and dosing adjustments are recommended in patients with moderate to severe liver insufficiency. Ivacaftor is not recommended for use in CF patients with a Child-Pugh (CP) class C liver disease, conferring severe hepatic dysfunction(20).

Ivacaftor has been associated with the development of cataracts in rat studies. Noncongenital cataracts have also been observed in children taking ivacaftor(21) Although there has been no confirmed causal relationship between ivacaftor and cataract development in human cases, as a precaution paediatric patients require baseline and regular ophthalmic review following ivacaftor initiation.

\subsubsection{Drug -drug Interactions}

As a consequence of polypharmacy in CF patients, the introduction of CFTR modulation necessitates particular attention to drug metabolism pathways and potential drug-drug interactions (DDI's). The most relevant drug metabolism pathway in CF involves oxidation by the cytochrome P450 (CYP450) enzyme family in hepatocytes, specifically the CYP3A4 and CYP2C9 subgroups(22).

Ivacaftor acts as a both a substrate and a weak inhibitor of the CYP3A4 enzymatic pathway. As such, ivacaftor has the potential for multiple DDI's with other medications exhibiting CYP3A4 enzymatic activity, often necessitating a dose change to ensure therapeutic drug 
levels(22). Several medications in the CF armamentarium affect CYP3A4 activity as either an enzyme inducer or enzyme inhibitor. A CYP3A4 inhibitor reduces enzyme activity such that plasma levels of the substrate (ivacaftor) increase. Conversely, CYP3A4 enzyme inducers decrease plasma substrate levels and consequently cause a subtherapeutic effect. An important example is the 'azole' antifungals such as itraconazole, used in the treatment of allergic bronchopulmonary aspergillosis (ABPA) in CF. Itraconazole is a strong CYP3A4 inhibitor which leads to increased ivacaftor exposure, and hence requires reduction of ivacaftor dose in order to use these medications simultaneously(23). Rifampicin is a strong CYP3A4 inducer and its concomitant administration with ivacaftor results in subtherapeutic modulator levels. Caution must also be taken with immunosuppressants such as tacrolimus and ciclosporin. These drugs are both CYP3A4 inhibitors and substrates, and the concurrent use of ivacaftor can increase plasma levels of immunosuppression, necessitating dose reductions in post-transplant CF patients(22).

\title{
2.1.3 Long-term results of ivacaftor therapy
}

Data from large US and UK registries analysing longer term ivacaftor use has suggested sustained clinical benefit. Over a four-year period in the UK cohort, absolute ppFEV 1 improved by $4.9 \%$ in the ivacaftor group compared to a $4.3 \%$ decline in matched nonivacaftor CF comparators. In addition, BMI improved by $1 \mathrm{~kg} / \mathrm{m}^{2}$ when compared with the non-ivacaftor group and rates of pulmonary exacerbation decreased(24).

\author{
Bessonova and colleagues showed that CF patients on ivacaftor have a statistically \\ significant lower risk of pulmonary exacerbation and hospitalisation when compared with \\ non-ivacaftor matched comparator groups in the US and UK. In US data, a reduction in
}


transplantation rate and mortality in comparison to a Phe508del propensity matched cohort was reported. In addition, a lower prevalence of Pseudomonas aeruginosa and Aspergillus species in sputum culture has been shown in ivacaftor cohorts following initiation of therapy(25). A study by Sawicki and colleagues in 2015 showed benefit from ivacaftor with sustained improvement in $\mathrm{ppFEV}_{1}$, weight and slower lung function decline when compared to non-ivacaftor Phe508del CF controls over a three-year period(26).

Currently we do not fully understand the potential longitudinal effects that effective CFTR modulation therapy will have for patients. Whilst there is some registry and clinical trial evidence of sustained improvement, this is not a universal signal. A recent study of ivacaftor treated patients in the Republic of Ireland suggested that rates of ppFEV ${ }_{1}$ decline did not significantly alter in adults who already had established pulmonary disease but improvements appeared to be sustained more readily in the younger CF cohort(27).

Ivacaftor monotherapy however has only been proven to be effective in a small proportion of CFTR mutations worldwide. When tested in patients with two Phe508del alleles, where there is substantially reduced amounts of epithelial CFTR protein, ivacaftor was not clinically effective, with no sustained improvement in $\mathrm{ppFEV}_{1}$ or sweat chloride after 16 weeks(28). This proved that CFTR potentiators alone could not achieve therapeutic effect in the majority of CF patients.

\subsection{Lumacaftor/Ivacaftor (Orkambi $\left.{ }^{\circledR}\right)$}

The lumacaftor/ivacaftor $\left(\mathrm{Orkambi}^{\circledR}\right)$ combination therapy combines a CFTR potentiator (ivacaftor) with a CFTR corrector (lumacaftor, VX-809). The lumacaftor entity works in 
synergy with ivacaftor to correct protein misfolding prior to its transport to the cell surface, where its resultant activity is enhanced by ivacaftor's effect on anion channel function. Together these compounds provide enhanced chloride transport(29). Lumacaftor/ivacaftor (lum/iva) has been tested in patients homozygous and heterozygous for the Phe508del mutation and was licensed for use in Phe508del homozygote patients in 2015. Interestingly, neither compound alone has proven to be efficacious in this CFTR mutation class and, although lumacaftor monotherapy exposed a modest reduction in sweat chloride, there was no improvement in lung function or respiratory symptom scores in Phe508del heterozygous patients after 28 days of treatment(30).

Phase 3 clinical trials conducted in 2013 and 2014, the TRAFFIC and TRANSPORT studies(31), showed sustained clinical benefit of lum/iva in CF Phe508del homozygotes over a 24-week period. A pooled analysis reported a 30-39\% decrease in pulmonary exacerbation rate, modest improvements in lung function (2.8\% $\mathrm{ppFEV}_{1}$ ) and $\mathrm{BMI}$ (mean improvement $\left.0.24 \mathrm{~kg} / \mathrm{m}^{2}\right)$, and statistically significant improvement in the respiratory domain of the CF specific quality of life tool (CFQ-R). Importantly however, the improvements in quality of life did not meet the minimal clinically important difference suggested for this measure.

Lum/iva has also proven to be efficacious in the younger paediatric CF population, with an improvement in lung clearance index $2.5\left(\mathrm{LCl}_{2.5}\right)$ and a $2.4 \%$ improvement in absolute ppFEV 1 in Phe508del homozygous children 6 to 11 years of age over a 24-week period(32). Longer term safety of lum/iva was assessed in the open label extension PROGRESS trial, spanning up to 120 weeks. This showed sustained benefit of the original primary outcome measures, with similar safety profiles, and a $42 \%$ slower rate of $\mathrm{ppFEV}_{1}$ decline when 
matched US CF registry controls(33). It should be noted however that the rate of decline in this control group was in excess of that reported in a similar cohort of controls from the US registry, which examined rate of decline in ivacaftor treated patients. Additionally, we should note that the comparison is between clinical trial participants and non-trial participants which may be a confounding factor even with the most rigorous propensity matching(26).

\subsubsection{Adverse events}

Unfortunately, despite this clinical effectiveness, tolerability of lum/iva has been limited by respiratory adverse events. The rate of serious adverse events in the lum/iva cohorts in the TRAFFIC and TRANSPORT trials(31) were around $17 \%$, with a therapy discontinuation rate of $4.2 \%$ versus $1.6 \%$ in the placebo group. The most frequently seen adverse events associated with lum/Iva in phase 3 clinical trials were observed within a few days of drug initiation and were predominantly respiratory in origin, including infective pulmonary exacerbation, dyspnoea, cough, increased sputum production and chest tightness(31). A study by TaylorCousar and colleagues(34) described an increased respiratory adverse event profile to lum/iva in CF patients with ppFEV 1 less than $40 \%$ predicted as compared to those with higher lung function. Dose reduction and close monitoring of up-titration was beneficial in this group and, as a result, no participants discontinued therapy. A number of clinical studies have also highlighted the potential for respiratory adverse events in patients with severe baseline lung function impairment(35). Current evidence would point to an off-target effect of lumacaftor reducing spirometric parameters(36). Similar decline in pulmonary function has been witnessed in healthy controls treated with lumacaftor(37). In this study, decline was attenuated by the use of bronchodilators, however this preventative strategy 
has not proven to be effective in all clinical reports, particularly in patients with severe lung disease(38).

Additional monitoring may be required for patients with higher CP classes of liver impairment, and liver function should be monitored closely following initiation of therapy. Similar to ivacaftor, cataract surveillance must be performed in younger CF populations. Lum/iva is not recommended for use peri-partum and in the post-partum period if breastfeeding(39).

\subsubsection{Drug-drug interactions}

The DDI's for lum/iva again originate predominantly from the hepatic CYP3A4 oxidation pathway. Lumacaftor is a strong CYP3A4 inducer and ivacaftor is a CYP3A4 substrate. The resulting effects of the lum/iva combination are therefore more complex and harder to predict than for ivacaftor alone and include reduction in efficacy of rifampicin, hormonal contraceptives, anti-epileptics and some classes of antidepressants(39). Patients must therefore be counselled regarding hormonal contraceptive issues before commencing CFTR modulator treatment and alternative methods are recommended.

If clinical status necessitates the prescription of CYP3A4 enzyme inducers or inhibitors alongside lum/iva or vice versa, dose adjustment strategies or alternative medication regimens should be instigated in order to combat these issues. Interestingly, given that lumacaftor is a CYP3A4 inducer and ivacaftor is a CYP3A4 substrate, when used in combination the exposure of ivacaftor is reduced by lumacaftor by up to $80 \%(40)$. To compensate for this reduced efficacy, ivacaftor dosing in dual CFTR therapy is increased from $150 \mathrm{mg}$ twice daily to $250 \mathrm{mg}$ twice daily. 


\subsection{Tezacaftor/ivacaftor (Symdeko ${ }^{\circledR}$ or Symkevi/Kalydeco ${ }^{\circledR}$ )}

Tezacaftor (VX-661) is the most recent CFTR corrector approved for use in CF modulator therapies. Since 2018, tezcaftor/ivacaftor (tez/iva) has been licensed for use in Phe508del homozygotes aged 12 years and above and for those with heterozygote Phe508del coupled with certain 'residual function' (RF) CFTR mutations(41).

Tezacaftor is a small molecule bound almost exclusively to plasma proteins. It is structurally similar to lumacaftor and is also metabolised via the CYP3A4 hepatic oxidation pathway. Unlike lumacaftor however, tezacaftor is not an inducer of the CYP3A4 enzyme and thus has less DDI's than its predecessor. Importantly, clinical trials have shown fewer respiratory adverse events than with lum/iva and better tolerability. The pharmacokinetic (PK) and clinical properties of tezacaftor and ivacaftor are shown in table 3.

As a CFTR corrector, tezacaftor acts to improve CFTR protein processing and trafficking, preventing early ribosomal protein degradation and increasing functional CFTR at the epithelial cell surface. Gating abnormalities also exist with Phe508del CFTR, which can be targeted by ivacaftor once the abnormal protein is expressed at the cell surface. Tezacaftor monotherapy has been shown in in vitro cell culture to improve cellular anion transport and this effect is almost doubled when acting in synergy with ivacaftor(42), providing proof of concept for its combined use in Phe508del mutations. 
Table 4 outlines the CFTR mutations that have shown response to tez/iva therapy(41), either

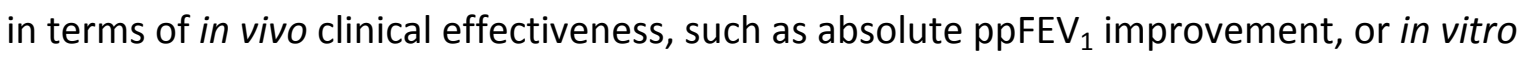
improvement of chloride transport in HBE cell studies.

\subsubsection{Phase 2 trials}

In 2018, Donaldson and colleagues published the first report on the clinical efficacy and safety of tez/iva safety in a phase 2 clinical trial(43). The treatment period was eight weeks, in Phe508del homozygotes and Phe508del/Gly551Asp heterozygotes already established on ivacaftor, with baseline $\mathrm{ppFEV}_{1}$ between 40 and $90 \%$. Incremental dosing of tezacaftor monotherapy and dual therapy with ivacaftor was studied. In the tez/iva study arms, results showed an acceptable adverse event profile, a reduction in sweat chloride of 6.04 and $7.02 \mathrm{mmol} / \mathrm{L}$, an increase in absolute $\mathrm{ppFEV}_{1}$ of 3.75 and $4.06 \%$, and an improvement in CFQR scores by 7.62 and 3.79 points in Phe508del homozygotes and heterozygotes respectively. The greatest within-group benefit was shown on tezacaftor 100mg daily in combination with ivacaftor $150 \mathrm{mg}$ twice daily, validating the optimal dosing schedule for subsequent phase 3 trials. Tezacaftor alone showed benefit to both lung function and sweat chloride, however there was no clear dose response pattern, perhaps due to small participant numbers in monotherapy groups.

\subsubsection{Phase 3 trials}

Two large, multicentre phase 3 trials have shown efficacy of tez/iva in both Phe508del homozygotes(44) and compound heterozygotes with a Phe508del allele coupled with a RF CFTR mutation(45). 


\subsubsection{EVOLVE}

The Evolve trial(44) was a phase 3, randomised, double-blind, multi-centre trial conducted over a 24-week period, evaluating the effectiveness of tez/iva in Phe508del homozygotes aged twelve years and above, with a baseline ppFEV 1 of $40-90 \%$. Tezacaftor was prescribed at $100 \mathrm{mg}$ once a day, with ivacaftor $150 \mathrm{mg}$ twice a day. 510 patients were recruited. Mean age was 26.3 years and mean baseline $\mathrm{ppFEV}_{1}$ was $60 \%$ predicted. The primary end point was absolute change in $\mathrm{ppFEV}_{1}$, and secondary measures included sweat chloride levels, pulmonary exacerbation rate, $\mathrm{BMI}$ and symptom change using CFQ-R.

A $4 \%$ absolute increase in $\mathrm{ppFEV}_{1}$ was observed in the tez/iva group, along with a $35 \%$ reduction in pulmonary exacerbations and a $10.1 \mathrm{mmol} / \mathrm{L}$ reduction in sweat chloride as compared with placebo. Improvements in BMI and CFQ-R were seen but were not statistically significant.

Although over $90 \%$ of patients in the tez/iva arm experienced mild adverse events, serious adverse events were significantly less (12.4\%) and drop-out rate was $2.8 \%$, all lower than in the placebo group. Most common adverse events included pulmonary exacerbation, cough, headache and nasopharyngitis, with the majority of these occurring less frequently in the tez/iva group compared to placebo. Importantly, when compared to lum/iva trial data, tez/iva was not associated with an increased incidence of respiratory adverse events or $\mathrm{ppFEV}_{1}$ decline post initiation of therapy.

\subsubsection{EXPAND}


The EXPAND trial(45) was a phase 3, randomised, double-blind, placebo controlled, crossover trial evaluating the efficacy of tez/iva in patients with one Phe508del and one CFTR mutation which conferred residual protein activity and was responsive to ivacaftor therapy in vitro (termed 'residual function' in this study). Patients in a complicated crossover design received either combination therapy with tez/iva, monotherapy with ivacaftor or placebo during two, eight-week time treatment periods separated by an eightweek washout. Eligibility criteria also included patients aged 12 years and above, baseline $\operatorname{ppFEV}_{1}$ between 40 and $90 \%$ and either a sweat chloride of over $60 \mathrm{mmol} / \mathrm{L}$ or documented sinopulmonary disease. Primary and secondary end points were similar to EVOLVE(44) and 25 specific CFTR mutations in combination with Phe508del were included in the study. Additional secondary endpoints for EXPAND included faecal elastase-1 and immunoreactive trypsinogen (IRT) levels, both markers of exocrine pancreatic function.

This study of 248 eligible CF patients confirmed that tez/iva was superior to ivacaftor alone in Phe508del/RF mutations. The CFTR correcting properties of tezacaftor on abnormal Phe508del CFTR protein enable enhancement of CFTR function not affected by ivacaftor CFTR anion channel potentiation alone. There was an absolute increase in ppFEV $\mathrm{F}_{1}$ of $6.8 \%$ in the tez/iva group compared to $4.7 \%$ in the ivacaftor arm, which was statistically significant. There was an 11.1 and 9.7-point improvement in the CFQ-R symptom score in tez/iva and ivacaftor arms respectively, statistically significant compared to placebo but not between therapy groups. Faecal elastase-1 and IRT levels showed a trend in favour of the treatment arms, although not statistically significant. This may suggest a pancreatic protective effect from tez/iva in CF patients with RF CFTR mutations(45). The degree of sweat chloride reduction in the ivacaftor arm of EXPAND was significantly lower than data shown in 
previous ivacaftor monotherapy trials. However, patients with RF mutations typically tend to have lower sweat chloride and milder disease phenotype. The baseline sweat chloride levels in the ivacaftor cohort of the original STRIVE study (ivacaftor monotherapy in those with a Gly551Asp gene) was $104.3 \mathrm{mmol} / \mathrm{L}$ as compared to 64.1 and 79.4 in the tez/iva and ivacaftor groups respectively in EXPAND. Following treatment these improved to 56.4, 54.6, and $74.9 \mathrm{mmol} / \mathrm{L}$ respectively.

Adverse events were similar in prevalence across all study groups and were comparable to the EVOLVE(44) study. $72 \%$ of patients on tez/iva experienced an adverse event, a higher rate than the ivacaftor group but lower than placebo. Around $58 \%$ were deemed as minor events and all were seen at a lower rate than placebo, including infective pulmonary exacerbation, cough, fatigue, haemoptysis and headache. There was no discontinuation of therapy in the tez/iva group and importantly, when compared with lumacaftor, there was no causal association between tezacaftor and respiratory adverse events in combination with ivacaftor. In addition, in 2018 Schwarz and colleagues reported successful transition to tez/iva in those patients unable to tolerate lum/iva(46).

\subsubsection{EXTEND}

The EXTEND trial(47) evaluates the longer-term safety and efficacy of tez/iva. Patients completing the EVOLVE trial were eligible to roll over into EXTEND to complete this 96-week open-label extension study and those patients on placebo were changed to tez/iva for the remaining study time frame. Interim data analysis from EXTEND after 48 weeks showed a sustained improvement in $\mathrm{ppFEV}_{1}$ after an additional 24 weeks of tez/iva, alongside an increase CFQ-R score and reduction in pulmonary exacerbation rate. 
A further two, phase 3 trials have shown tez/iva to have no meaningful clinical improvement in heterozygote patients with Phe508del and a minimal function (MF) mutation or patients with both Phe508del and Gly551Asp alleles(42). These intriguing results merit further discussion. The lack of efficacy in compound heterozygotes for Phe508del and a nonresponsive (MF) mutation indicates that tez/iva does not confer sufficient modulating activities to afford clinical benefit when targeting only one Phe508del allele. The lack of response in patients heterozygous for Phe508del and a gating mutation was similarly of note, particularly as the results appeared in contrast to earlier phase 2 work. Although we await formal publication of the trial results, which may better explain these findings, these results raise questions about the additional potency of combination therapy to achieve clinical efficacy in patients who are already receiving effective CFTR modulation. These data imply that within some mutations we may have already achieved the ceiling of effect of CFTR modulation.

The EVOLVE(44) and EXPAND(45) trials established safety and efficacy of tez/iva in CF patients aged 12 years or above with Phe508del homozygosity or compound heterozygotes with Phe508del and RF mutations. Data from a phase 3 study of tez/iva safety and efficacy in a younger CF patient cohort with comparable genetic profiles have recently been published(48). This two-part study, similar in methodology to the previous KIWI study for ivacaftor(19), investigated pharmacokinetics (PK), tolerability, adverse events and clinical efficacy of weight-adjusted tez/iva dosing in 83 CF patients aged six to eleven years of age. Demographic data in this cohort showed well-preserved mean baseline $\mathrm{ppFEV}_{1}$ of around $90 \%$ and mean sweat chloride of $99.1 \mathrm{mmol} / \mathrm{L}$. Tez/iva was found to have a satisfactory PK and adverse event profile in this patient group. Minor adverse events were seen in the 
majority (92.9\%) but only $4.3 \%$ of participants reported a serious adverse event, most commonly pulmonary exacerbation. Elevation of liver transaminases was seen in a small number of patients, resolving without need for drug discontinuation in all but one case. Adverse events in this age group occurred in a similar pattern to older patient cohorts(45). Although no placebo group was enrolled, results show a sustained reduction in sweat chloride over 24 weeks, ppFEV 1 stability and an improvement in CFQ-R scores.

Walker and colleagues have recently reported the results of a phase 3 tez/iva trial in children aged 6 to 11 years, the first tez/iva data in this cohort(49). Phe508del homozygotes and Phe508del heterozygotes with an eligible RF mutation were included. Weight adjusted tez/iva showed good safety and tolerability in this group, with improvements in sweat chloride similar to that of older children and adults. Minor improvements in CFQ-R were seen in this group despite relatively low baseline respiratory symptom burden, however there was no improvement seen in $\mathrm{ppFEV}_{1}$, probably reflecting the higher baseline lung function in this cohort.

\subsubsection{Adverse events and drug-drug interactions with tez/iva}

The above combined trial data consistently showed tez/iva to have a more favourable side effect profile than its predecessor lum/iva. Respiratory adverse events were less frequent in the EVOLVE and EXPAND trials in treatment arms compared with placebo, including at initiation of treatment and for those with lower levels of baseline lung function. No participants withdrew as a result of respiratory symptoms, in contrast to lum/iva phase 3 trials. 
Unlike lumacaftor, tezacaftor is not a CYP3A4 enzyme inducer and therefore it accounts for less DDI's with other important CF medication. Tezacaftor is however a CYP3A4 substrate. The implications of this require modification of dosing when tez/iva is co-administered with CYP3A4 enzyme inducers such as rifampicin, and enzyme inhibitors such as azole antifungals. Hormonal contraception can be used reliably with tez/iva. Both tezacaftor and ivacaftor are weak inhibitors of the P-glycoprotein (P-gp) pathway, an important process in drug absorption across cell membranes. As a result, concomitant use of tez/iva with sensitive P-gp substrates, such as digoxin and some immunosuppressants, requires dose adjustments and closer monitoring of plasma levels(23).

Elevation of liver transaminases have been observed in all tez/iva phase 3 trials(44)(45) in a very small percentage of patients (0.6-2\%) but all were $\leq 8 \mathrm{x}$ ULN, thus classed as mild to moderate, and none led to drug discontinuation.

\title{
3.0 Pregnancy and CFTR modulation
}

The rate of pregnancy in CF is rising and, with an ageing CF population and more women reaching reproductive age, there is potential for this to increase further. Since over $80 \%$ of CF patients may ultimately be suitable for CFTR modulation therapy, it is important to understand the implications of these drugs in pre, peri and post-natal periods.

\author{
No teratogenic effects have been recognised with ivacaftor or lum/iva in the first two \\ trimesters of pregnancy in animal studies. Due to the lack of human data however, their \\ avoidance in pregnancy is recommended(50). There is debate surrounding the use of CFTR \\ modulators and clinical decline during pregnancy and there have been successful CF
}


pregnancies whilst taking CFTR modulators in this context(51). A multicentre survey by Nash and colleagues published earlier this year observed 13 out of 16 successful pregnancies in women taking ivacaftor, with no foetal abnormalities. There were no maternal deaths and a $12.5 \%$ miscarriage rate was observed in this small cohort(52).

Ivacaftor has been shown to be present in placental and foetal umbilical cord blood. In addition, ivacaftor is present in low levels in breast milk(53), the significance of which remains uncertain, however breastfeeding on ivacaftor is currently not recommended.

\subsection{Tez/iva vs lum/iva "head to head"}

Phase 3 trials of tez/iva(44)(45) and lum/iva(31) have proven clinical efficacy of both CFTR modulator compounds in terms of absolute $\mathrm{ppFEV}_{1}$, pulmonary exacerbation rate and nutritional parameters. Lung function benefit from tez/iva appears marginally greater than seen with lum/iva, although compared with ivacaftor monotherapy, improvements in absolute $\mathrm{ppFEV}_{1}, \mathrm{BMI}$ and sweat chloride with both combinations are modest. Comparative data from pivotal ivacaftor, lum/iva and tez/iva trials are shown in table 5.

Respiratory adverse events with lumacaftor have significantly limited its use, particularly in patients with lower baseline lung function. Lum/iva has been shown to cause a $10 \%$ reduction in $\mathrm{ppFEV}_{1}$ even in healthy volunteers(55). Current evidence suggests tez/iva superiority in terms of tolerability and drug discontinuation rates(46). Significant respiratory adverse events have not been observed with tezacaftor thus far, nor in additional CFTR correctors in triple therapy preliminary data. 
Due to the above factors, in particular its superiority in relation to DDI's, tez/iva is beginning to be utilised as a first line CFTR modulator for Phe508del homozygotes, as opposed to second line following lum/iva failure. Of note, there have been no trials testing efficacy of tez/iva in patients with lower lung function and this will be important to determine accurate tolerability and adverse event data in CF cohorts with more severe lung disease.

In vitro studies have shown that ivacaftor can diminish the CFTR correction function of both lumacaftor and tezacaftor when used in dual combination in Phe508del homozygotes(56)(57). This may assist to explain the difference in clinical efficacy when compared to ivacaftor monotherapy.

\subsection{Triple modulation therapy}

There is currently no available modulator treatment for patients with rarer CFTR mutations such as minimal function (MF) mutations. These encompass either a "nonsense" mutation that produces no normal CFTR protein, e.g. Gly542X, or a CFTR mutation that results in severely defective CFTR processing, such as N1303K, and hence has no response to CFTR modulation. Around $30 \%$ of CF patients are heterozygous for Phe508del and a MF mutation(58), with only one potentially modifiable disease-responsive allele. Triple therapy uses next generation CFTR correctors, VX-445 and VX-659, in combination with previous dual therapy (tez/iva) to maximise Phe508del CFTR function with an attempt to achieve high levels of CFTR function despite one unresponsive allele(59)(60).

VX-445 in combination with tez/iva has been reported in a phase II trial(60) to result in improvement in absolute ppFEV1 of 13.8 and 11\% in Phe508del/MF heterozygotes and 
Phe508del homozygotes respectively. In addition, a significant reduction in sweat chloride and improvement in CFQ-R scores were seen in both genotype groups, to a much higher degree than observed differences with dual therapy. Minor adverse respiratory events were common, with a profile not dissimilar to the preceding tez/iva trials. A $4 \%$ rate of serious adverse events was observed in the VX-445 triple therapy group, which consisted of pulmonary exacerbation and distal intestinal obstruction syndrome (DIOS). Three patients discontinued therapy.

\subsection{Expert Opinion}

The development of CFTR modulators has been correctly heralded as a major advance in CF and has resulted in meaningful clinical benefits for large numbers of patients. Key questions however persist in the ongoing goal to provide effective therapies targeted at the basic default in CF for all patients. With relation to tez/iva, the current evidence would support its use in Phe508del homozygote patients, and those carrying one Phe508del gene and one 'residual function' mutation. More importantly, the five-year horizon suggests that tez/iva will have a fundamental role as the basis of future CF therapies but with its activity augmented by additional agents. Currently trials are ongoing using tez/iva as the basis for 'triple therapy' with the addition of a further 'corrector' molecule to increase CFTR expression at the cell surface. There is also interest in using these compounds in addition to a compound which enhances translation of CFTR protein, the so-called class of 'CFTR amplifiers.'

\subsection{Unanswered questions}

With relation to tezacaftor/ivacaftor, some of the key unanswered questions are; 


\subsubsection{What are the long-term effects?}

We currently do not fully know the long-term impact of CFTR modulation. Longer studies including observational registry reports are necessary to establish whether dual combination therapy will have a true disease modifying impact with reduction in CFTR related complications and disease progression.

\subsubsection{When should we initiate therapy?}

It appears rational to suggest that earlier commencement of therapy before pathological damage occurs may be beneficial, but currently we do not have clear evidence to recommend when to start.

\subsubsection{Can we explain differential effects or predict which patients will respond to therapy beyond CFTR genotype?}

Research into the use of intestinal organoids to predict in vitro response to CFTR modulators is underway and could represent a method by which individualised patient response to modulation therapy can be assessed, independent of genetic eligibility. This will be covered in greater detail later in this review.

\subsubsection{How can adherence to these therapies be maximised?}

Alongside initiation of CFTR modulation, we must pay attention to treatment adherence and withdrawal of conventional CF maintenance therapies. Hubert et al reported a significant reduction in established CF maintenance treatments in a CF cohort two years following ivacaftor initiation(61). However, despite clinical benefit to patients, we know 
that compliance with ivacaftor is suboptimal and has been estimated to be as low as $61 \%(62)$. The importance of adherence is highlighted by the reports of 'ivacaftor withdrawal syndrome' where patients sustained a dramatic clinical decline as a result of the abrupt cessation of CFTR modulator therapy(63). All efforts to understand and improve adherence need to be harnessed to ensure the encouraging initial data regarding CFTR modulation is realised into the future.

\subsubsection{Is there a role for withdrawal of other treatments if successful CFTR modulation is in place?}

CF patients have a high treatment burden, and often struggle fitting demanding treatment regimens into their daily lives. All the trials so far have involved patients already on the full panoply of conventional CF therapies (including nebulised antibiotics and mucolytics) and have specifically required no elective changes to background regimens. If patients are more stable, will the additional effects of these be enough to offset the costs and burdens of taking them? These are hard questions to answer with formal trials. This question however appears to be important to patients and care providers. Gifford and colleagues presented results of a 2018 survey of CF patients, their families and CF healthcare providers in the US exploring the potential of a trial which would reduce medication burden following initiation of CFTR modulator therapy. Over $80 \%$ of patients and $95 \%$ of care providers supported the idea of ' withdrawal of therapy' trials. Interestingly almost a quarter of patients surveyed who were taking ivacaftor or tez/iva had already discontinued some therapy(64). 


\title{
6.1.6 How can we conduct studies to examine whether one CFTR modulator is
} superior to another?

Moving forwards in the development of CFTR modulation therapy, clinical trials providing a direct comparison of the efficacy of available CFTR modulators may be informative, particularly in those patients with more advanced lung disease and comorbidities, and in whom a more in-depth review of DDI's and potential adverse events is warranted. In vitro cell models using intestinal organoids to assess modulator response may also add to our overall understanding of the differences in modulator efficacy and individual clinical outcomes.

\subsubsection{How do we ensure equitable access to CFTR modulators?}

This is perhaps globally the greatest challenge for high cost medications. Access to medications where this therapy is licensed has differed according to funding arrangements for health care. With relation to tez/iva there is an additional complexity in that licensed indications for therapy differ in the EU and the US for the subset of patients who are heterozygote for Phe508del and a 'residual function' mutation. In the US the FDA approved 12 additional mutations based on basic science data, previous translational work and safety data. Ultimately assuring equitable access for all patients who would benefit for a therapy has to be the goal for the CF clinical community.

\begin{abstract}
As we have seen from the ivacaftor registry studies, highly effective CFTR modulation is not sufficient to reverse established airway disease or entirely halt lung function decline. There will therefore continue to be a need for medications aimed at the secondary consequences of CFTR dysfunction, including anti-inflammatory, anti-infective and mucolytic treatments.
\end{abstract}




\begin{abstract}
CF patients experience a chronic high inflammatory burden, with increased quantity of inflammatory mediators such as macrophages, neutrophils and interleukins (IL) in CF airways, contributing to progressive lung disease. Anti-inflammatory compounds have gained particular interest in recent years, with lenabasum and acebilustat yielding promising phase II results(65).
\end{abstract}

\title{
6.2 CFTR modulation in early CF life
}

Many of the irreversible pathological processes in cystic fibrosis begin in early life. Exocrine pancreatic insufficiency, gastrointestinal dysfunction and subsequent nutritional impairment in postnatal and infant periods contributes to increased prevalence of lung infections and earlier disease progression. It is important therefore to study the effect of CFTR modulation in early life, with the potential thereby to prevent or halt CFTR-mediated disease progression. Sun and colleagues(66) have studied the effect of ivacaftor on epithelial cells of ferret CF models both in utero and early postnatal life. Animal models in CF have significant variation in disease expression, and ferrets are particularly susceptible to pancreatic and gastrointestinal CF disease. In ferret models, in utero, ivacaftor was shown to reduce the prevalence of meconium ileus following birth. We are a long way from providing these treatments to unborn children, but early commencement of highly effective modulators, with sweat chloride reduced to levels similar to those seen in non-CF population, suggest that the long-term sequelae of CF could be substantially reduced.

\subsection{CFTR modulation in advanced lung disease} Ivacaftor and lum/iva have been shown to be efficacious and well tolerated in initial clinical trials for patients with mild to moderate lung disease (i.e. baseline ppFEV ${ }_{1}$ of above $40 \%$ ). Patients with more severe impairment of lung function are however routinely excluded 
from clinical trials, and there is consequently little in the way of trial data on efficacy and tolerability in this group, despite the potential for more urgent need of such therapies. Ivacaftor has been shown to provide both clinical benefit and acceptable tolerability in patients with a baseline ppFEV ${ }_{1}$ of below $40 \%(17)$. However, lum/iva appears to be less well tolerated in CF cohorts with more advanced lung disease, which appears to be caused by an 'off-target' effect of lumacaftor. Several clinical studies have shown a higher prevalence of respiratory adverse events and drug discontinuation rates with lum/iva in CF cohorts with severe lung disease $\left(\mathrm{FEV}_{1}<40 \%\right)$ than had been reported in the clinical trials(35)(38). These patients may require closer monitoring at initiation of therapy and a graduated approach to dosing(34).

To date there have been no similar reports of respiratory adverse events for tez/iva, despite it now being prescribed on managed access programmes for a wide range of lung function and available in a large number of geographical locations. Tez/iva phase 3 trials included a small percentage of patients with baseline $\mathrm{ppFEV}_{1}$ of $<40 \%$, with similar improvements in lung function as their less severe lung disease counterparts, and no disadvantage in terms of adverse events(44)(45). However, tez/iva has not been specifically assessed in a larger cohort of patients with severe lung disease, and there have not been clinical reports of the high discontinuation rates seen with lum/iva. This specific advantage of tezacaftor over lumacaftor is one of the factors that led to its inclusion in triple modulation therapy.

\subsection{CFTR modulation following solid organ transplantation}

The use of CFTR modulators in CF patients following organ transplantation is contentious and an area that currently lacks clinical data. There is a fine balance between the risk of drug interactions with immunosuppressant therapy, subsequent implications for organ 
rejection or drug toxicity, and the potential benefits of CFTR modulation on native CF organs. The use of CFTR modulation following double lung transplantation is currently unlicensed, although inferred benefits despite lack of CF lung disease may include improvements in nutritional status, sinus disease and quality of life. The use of CFTR modulation has been reported successfully following extra-pulmonary solid organ transplantation in CF. In 2018, Choucane and colleagues reported two cases of lum/iva use at different stages following liver transplantation due to deterioration in lung function(67). Tacrolimus dose was increased prior to commencement of lum/iva in one case, which resulted in greater stability of tacrolimus levels thereafter. Graduated dosing of lum/iva was used in both patients. In both cases it was proven possible to maintain satisfactory levels of immunosuppression alongside gaining some benefit in absolute $\mathrm{ppFEV}_{1}$ with lum/iva. Voriconazole was used concomitantly in both patients and, unsurprisingly, proved challenging to maintain satisfactory plasma levels due to the strong CYP3A4 inducing activity of lumacaftor.

We are now beginning to see an ageing CF population and, as a consequence, CF patients will face advancing CFTR-related comorbidity and organ dysfunction, alongside non-CFTR ageing complications. There is also likely to be an increasing availability of CFTR modulators moving forwards. The consequence of these factors may result in increasing organ transplantation rates and a natural progression towards the use of CFTR modulation posttransplant, thus an important area for further research. If clinical benefit is shown, it may be possible to use CFTR modulation safely in post-transplant patients with more intensive drug monitoring(68). 


\subsection{The future of CFTR therapy}

\subsubsection{Triple Therapy}

VX-445 and VX-659 in combination with tez/iva are currently in phase III trials. Preliminary results show an improvement in absolute $\mathrm{ppFEV}_{1}$ in Phe508del homozygotes and Phe508del/MF heterozygote patient groups for both compounds, with VX-445 showing slight superiority compared to VX-659. A decision was made in May 2019 by Vertex Pharmaceuticals to take VX-445 forward preferentially to phase III open label and if successful, these trials will pave the way for creating CFTR modulator therapy opportunities for almost $90 \%$ of CF patients worldwide(69). Triple modulation therapy is predicted to have a major influence on the future of CF treatment and survival.

The existence of multiple class effects on CFTR protein within genotypes and variable clinical response introduces inaccuracies when determining eligibility and predicting response to CFTR modulators by mutation class alone(70). In addition, there remains a proportion of CF patients with rarer CFTR mutations with no option for trials of potential disease-modifying drugs. The evidence base to provide these CF cohorts with CFTR modulation is predictably small due to lower patient numbers and resultant lack of inclusion in clinical trials.

\subsubsection{In vitro and organoid models}

Recently greater interest has been focussed on testing patient's own tissue in in vitro cellular CFTR models in order to predict in-vivo clinical response in individual patients, using intestinal epithelial organoid cell models cultured from rectal mucosa. The ability of the organoid cells to swell with forskolin (a cyclic adenosine monophosphate activator - cAMP) is dependent upon degree of CFTR function. Increased forskolin-induced swelling correlates with better CFTR function and vice versa(71). In this way, individual CFTR dysfunction and 
improvement with modulator therapy can be analysed. Organoid swelling has been found to be proportional to sweat chloride and although in vitro clinical response may not fully translate into clinical efficacy, this method is promising and may lead to individualised prediction of CFTR modulator eligibility and response(72). This process could be used to test in vitro combinations of CFTR correctors, potentiators and amplifiers, from the wide range of pharma companies now active in this area. The method would allow head to head comparisons of different combinations and dose optimisation prior to clinical trials. Furthermore, within the next decade we would anticipate that an individual CF patient, either with rarer genotype or showing poor response to first line CFTR modulation, might have an individualised assessment of CFTR corrector/potentiator combinations, to select an individual treatment regimen/dosing optimised to their specific CFTR genes and modifiers.

\subsection{Conclusion}

CFTR modulators represent the most important advance in any respiratory disease in the last twenty years. They are the first class of treatment aimed at restoring the basic defect in CF and herald a new era in individualised therapy. They have replaced gene therapy as the hope, for many patients, of a "cure" for CF. Trial data so far has shown that tez/iva is the best tolerated and most effective of the two combination therapies currently available. The simpler DDI's and lack of initial chest tightness are the predominant advantages of tez/iva when compared to lum/iva, since the clinical effectiveness of each is similar. Importantly however, tez/iva looks like it will form the backbone of exciting triple combination therapies with the potential to provide levels of CFTR correction similar to that seen with ivacaftor in Gly551Asp patients. For older patients with established disease, the community hopes this will lead to disease stabilisation and reduction in symptoms for the majority of patients. For 
younger patients, they may be able to avoid the worst of the long-term consequences of chronic airway obstruction and infection, and CF in another 20 years may appear quite different from today.

\section{Information Resources}

- Clinical trial details can be found at https://clinicaltrials.gov

- Up to date information regarding tez/iva and triple therapy trial data can be found at https://investors.vrtx.com.

- Cystic fibrosis general information and registry data can be found at www.cysticfibrosis.org.uk (UK) and https://www.cff.org (US) 


\section{Funding}

This paper was not funded

\section{Declaration of interest}

A Horsley reports personal fees from Vertex Pharmaceuticals Incorporated, during the conduct of the study, personal fees from Celtaxsys, personal fees from Chiesi, personal fees from Boehringer Ingelheim, grants from NIHR, grants from Cystic Fibrosis Foundation, grants from Cystic Fibrosis Trust, grants from Gilead, grants from Proteostasis. P Barry Has been investigator on clinical trials sponsored by Vertex pharmaceuticals involving the use of tezacaftor/ivacaftor. Has received speaker/consultancy fees from Vertex pharmaceuticals Itd. Member of the organizing committee of an educational conference which has received sponsorship from Vertex pharmaceuticals, Chiesi pharmaceuticals, Gilead. The authors have no other relevant affiliations or financial involvement with any organization or entity with a financial interest in or financial conflict with the subject matter or materials discussed in the manuscript apart from those disclosed.

\section{Reviewer disclosures}

A peer reviewer on this manuscript has disclosed that they are a scientific consultant for Vertex Pharmaceuticals. Peer reviewers on this manuscript have no other relevant financial or other relationships to disclose. 


\section{References}

Papers of special note have been highlighted as:

* of interest

** of considerable interest

1. Chapman S, Robinson G, Stradling J. Cystic fibrosis. Oxford Handbook of Respiratory Medicine. 3rd Edition. Oxford University Press. 2014;p210.

2. Cutting GR. Cystic fibrosis genetics: from molecular understanding to clinical application. Nat Rev Genet. 2015;16(1):45-56.

3. Soe K, Gregoire-Bottex MM. A rare CFTR mutation associated with severe disease progression in a 10-year-old Hispanic patient. Clin case reports. 2017;5(2):139-44.

4. Veit G, Avramescu RG, Chiang AN et al. From CFTR biology toward combinatorial pharmacotherapy: expanded classification of cystic fibrosis mutations. Mol Biol Cell. 2016;27(3):424-33.

5. Merlo CA, Boyle MP. Modifier genes in cystic fibrosis lung disease. J Lab Clin Med. 2003;141(4):237-41.

6. Rowntree RK, Harris A. The Phenotypic Consequences of CFTR Mutations. Ann Hum Genet. 2003;67(5):471-85.

7. Boyle MP, De Boeck K. A new era in the treatment of cystic fibrosis: correction of the underlying CFTR defect. Lancet Respir Med. 2013;1(2):158-63.

8. Ramsden D, Carr S. Cystic Fibrosis Trust Annual Report [Internet] 2018;p8-77. [cited 2018 Aug 28]. Available from: http://www.cysticfibrosis.org.uk/the-work-we-do/ukcf-registry/reporting-and-resources.

9. Cystic Fibrosis Foundation. Patient Registry | CF Foundation [Internet]. 2018 [cited 2019 Jul 25]. Available from: https://www.cff.org/Research/Researcher- 


\section{Resources/Patient-Registry/.}

10. De Boeck K, Zolin A, Cuppens $\mathrm{H}$ et al. The relative frequency of CFTR mutation classes in European patients with cystic fibrosis. J Cyst Fibros. 2014;13(4):403-9.

11. Yu H, Burton B, Huang C-J et al. Ivacaftor potentiation of multiple CFTR channels with gating mutations. J Cyst Fibros. 2012;11(3):237-45.

12. Elborn JS. Fixing cystic fibrosis CFTR with correctors and potentiators. Off to a good start. Thorax. 2012;67(1):4-5.

13. Van Goor F, Hadida S, Grootenhuis PDJ et al. Rescue of CF airway epithelial cell function in vitro by a CFTR potentiator, VX-770. Proc Natl Acad Sci U S A. 2009;106(44):18825-30.

*14. Ramsey BW, Davies J, McElvaney NG et al. A CFTR Potentiator in Patients with Cystic Fibrosis and the G551D Mutation. N Engl J Med. 2011;365(18):1663-72.

*Ivacaftor phase 3 trial data.

15. Davies JC, Wainwright CE, Canny GJ et al. Efficacy and Safety of Ivacaftor in Patients Aged 6 to 11 Years with Cystic Fibrosis with a G551D Mutation. Am J Respir Crit Care Med. 2013;187(11):1219-25.

16. McKone EF, Borowitz D, Drevinek P et al. Long-term safety and efficacy of ivacaftor in patients with cystic fibrosis who have the Gly551Asp-CFTR mutation: a phase 3, openlabel extension study (PERSIST). Lancet Respir Med. 2014;2(11):902-10.

17. Barry P, Plant B, Nair A et al. Effects of ivacaftor in patients with cystic fibrosis who carry the G551D mutation and have severe lung disease. Chest. 2014;146(1):152-8.

18. Moss RB, Flume PA, Elborn JS et al. Efficacy and safety of ivacaftor in patients with cystic fibrosis who have an Arg117His-CFTR mutation: a double-blind, randomised controlled trial. Lancet Respir Med. 2015;3(7):524-33. 
19. Davies JC, Cunningham S, Harris WT et al. Safety, pharmacokinetics, and pharmacodynamics of ivacaftor in patients aged $2-5$ years with cystic fibrosis and a CFTR gating mutation (KIWI): an open-label, single-arm study. Lancet Respir Med. 2016;4(2):107-15.

20. Vertex. Product monograph, Kalydeco. [Internet]. Vertex 2012 [cited 2019 July 26]. Available from https://pi.vrtx.com/files/uspi_ivacaftor.pdf.

21. McColley SA. A safety evaluation of ivacaftor for the treatment of cystic fibrosis. Expert Opin Drug Saf. 2016;15(5):709-15.

22. Jordan CL, Noah TL, Henry MM. Therapeutic challenges posed by critical drug-drug interactions in cystic fibrosis. Pediatr Pulmonol. 2016;51(S44):S61-70.

23. Garg V, Shen J, Li C et al. Pharmacokinetic and Drug-Drug Interaction Profiles of the Combination of Tezacaftor/Ivacaftor. Clin Transl Sci. 2019;12(3):267-75.

24. Volkova N, Moy K, Evans J et al. Disease progression in patients with cystic fibrosis treated with ivacaftor: Data from national US and UK registries. J Cyst Fibros;(in press). 2019.

25. Bessonova L, Volkova N, Higgins M et al. Data from the US and UK cystic fibrosis registries support disease modification by CFTR modulation with ivacaftor. Thorax. 2018;73(8):731-40.

26. Sawicki GS, McKone EF, Pasta DJ et al. Sustained Benefit from Ivacaftor Demonstrated by Combining Clinical Trial and Cystic Fibrosis Patient Registry Data. Am J Respir Crit Care Med. 2015;192(7):836-42.

27. Kirwan L, Fletcher G, Harrington M et al. Longitudinal Trends in Real-World Outcomes after Initiation of Ivacaftor. A Cohort Study from the Cystic Fibrosis Registry of Ireland. Ann Am Thorac Soc. 2019;16(2):209-16. 
28. Flume PA, Liou TG, Borowitz DS et al. Ivacaftor in Subjects With Cystic Fibrosis Who Are Homozygous for the F508del-CFTR Mutation. Chest. 2012;142(3):718-24.

29. Brewington JJ, McPhail GL, Clancy JP. Lumacaftor alone and combined with ivacaftor: preclinical and clinical trial experience of F508del CFTR correction. Expert Rev Respir Med. 2016;10(1):5-17.

30. Clancy JP, Rowe SM, Accurso FJ et al. Results of a phase lla study of VX-809, an investigational CFTR corrector compound, in subjects with cystic fibrosis homozygous for the F508del-CFTR mutation. Thorax. 2012;67(1):12-8.

*31. Wainwright CE, Elborn JS, Ramsey BW et al. Lumacaftor-Ivacaftor in Patients with Cystic Fibrosis Homozygous for Phe508del CFTR. N Engl J Med. 2015;373(3):220-31.

*Lum/iva phase 3 trial data.

32. Ratjen F, Hug C, Marigowda G et al. Efficacy and safety of lumacaftor and ivacaftor in patients aged 6-11 years with cystic fibrosis homozygous for F508del-CFTR : a randomised, placebo-controlled phase 3 trial. Lancet Respir Med. 2017;5(7):557-67.

33. Konstan MW, McKone EF, Moss RB et al. Assessment of safety and efficacy of longterm treatment with combination lumacaftor and ivacaftor therapy in patients with cystic fibrosis homozygous for the F508del-CFTR mutation (PROGRESS): a phase 3, extension study. Lancet Respir Med. 2017;5(2):107-18.

34. Taylor-Cousar JL, Jain M, Barto TL et al. Lumacaftor/ivacaftor in patients with cystic fibrosis and advanced lung disease homozygous for F508del-CFTR. J Cyst Fibros. $2018 ; 17(2): 228-35$.

35. Popowicz N, Wood J, Tai A et al. Immediate effects of lumacaftor/ivacaftor administration on lung function in patients with severe cystic fibrosis lung disease. J Cyst Fibros. 2017;16(3):392-4. 
36. Jones AM, Barry PJ. Lumacaftor/ivacaftor for patients homozygous for Phe508delCFTR: should we curb our enthusiasm? Thorax. 2015;70(7):615-6.

37. Marigowda G, Liu F, Waltz D. Effect of bronchodilators in healthy individuals receiving lumacaftor/ivacaftor combination therapy. J Cyst Fibros. 2017;16(2):246-9.

38. Hubert D, Chiron R, Camara B et al. Real-life initiation of lumacaftor/ivacaftor combination in adults with cystic fibrosis homozygous for the Phe508del CFTR mutation and severe lung disease. J Cyst Fibros. 2017;16(3):388-91.

39. Talamo Guevara M, McColley SA. The safety of lumacaftor and ivacaftor for the treatment of cystic fibrosis. Expert Opin Drug Saf. 2017;16(11):1305-11.

40. Vertex Guidance and Patient Support (GPS). Drug Interactions | ORKAMBI ${ }^{\circledR}$ (lumacaftor/ivacaftor) [Internet]. Vertex. 2018 [cited 2019 Jul 26]. Available from: https://www.orkambihcp.com/drug-interactions.

41. Lommatzsch ST, Taylor-Cousar JL. The combination of tezacaftor and ivacaftor in the treatment of patients with cystic fibrosis: clinical evidence and future prospects in cystic fibrosis therapy. Ther Adv Respir Dis. 2019;13:1753466619844424.

42. Sala MA, Jain M. Tezacaftor for the treatment of cystic fibrosis. Expert Rev Respir Med. 2018;12(9):725-32.

43. Donaldson SH, Pilewski JM, Griese M et al. Tezacaftor/Ivacaftor in Subjects with Cystic Fibrosis and F508del/F508del-CFTR or F508del/G551D-CFTR. Am J Respir Crit Care Med. 2018;197(2):214-24.

*44. Taylor-Cousar JL, Munck A, McKone EF et al. Tezacaftor-Ivacaftor in Patients with Cystic Fibrosis Homozygous for Phe508del. N Engl J Med. 2017;377(21):2013-23. *Tez/iva phase 3 trial data in Phe508del homozygotes.

*45. Rowe SM, Daines C, Ringshausen FC et al. Tezacaftor-Ivacaftor in Residual-Function 
Heterozygotes with Cystic Fibrosis. N Engl J Med. 2017;377(21):2024-35.

*Tez/iva phase 3 trial data in Phe508del heterozygotes with a 'residual function' CFTR mutation.

46. Schwarz C, Sutharsan S, Epaud R et al. Safety, Efficacy, and Tolerability of Tezacaftor/Ivacaftor in Cystic Fibrosis Patients Who Previously Discontinued Lumacaftor/Ivacaftor Due To Respiratory Adverse Events: A Randomized, DoubleBlind, Placebo-Controlled Phase 3b Study. Pneumologie. 2019;73(S01)p.376.

47. Flume PA, Owen CA, Brown CD et al. Long-term Safety and Efficacy of Tezacaftor/Ivacaftor in Patients With Cystic Fibrosis Homozygous for F508del-CFTR or Heterozygous for F508del and a Residual Function Mutation: First Interim Analysis Results of a Phase 3, Open-label, Rollover Study. AJRCCM. 2018;197:A7807.

48. Walker S, Flume P, McNamara J et al. A phase 3 study of tezacaftor in combination with ivacaftor in children aged 6 through 11 years with cystic fibrosis. J Cyst Fibros. 2019;18(5):708-713.

49. Walker S, Flume P, McNamara J et al. A phase 3 study of tezacaftor in combination with ivacaftor in children aged 6 through 11 years with cystic fibrosis. J Cyst Fibros. 2019;18(5):708-13.

50. Kroon MAGM, Akkerman-Nijland AM, Rottier BL et al. Drugs during pregnancy and breast feeding in women diagnosed with Cystic Fibrosis - An update. J Cyst Fibros. $2018 ; 17(1): 17-25$.

51. Trimble A, McKinzie C, Terrell M et al. Measured fetal and neonatal exposure to Lumacaftor and Ivacaftor during pregnancy and while breastfeeding. J Cyst Fibros. 2018;17(6):779-82.

52. Nash EF, Brokaar E, Casey R et al. WS12-2-1 Pregnancy outcomes in women with 
cystic fibrosis on ivacaftor - an international survey. J Cyst Fibros. 2019;18:S22.

53. Kaminski R, Nazareth D. Letter to the Editor A successful uncomplicated CF pregnancy while remaining on Ivacaftor. J Cyst Fibros. 2016;15:133-4.

54. Boyle MP, Bell SC, Konstan MW et al. A CFTR corrector (lumacaftor) and a CFTR potentiator (ivacaftor) for treatment of patients with cystic fibrosis who have a phe508del CFTR mutation: a phase 2 randomised controlled trial. Lancet Respir Med. 2014;2(7):527-38.

55. Habib A-RR, Kajbafzadeh M, Desai S et al. A Systematic Review of the Clinical Efficacy and Safety of CFTR Modulators in Cystic Fibrosis. Sci Rep. 2019;9(1):7234.

56. Veit G, Avramescu RG, Perdomo D et al. Some gating potentiators, including VX-770, diminish F508-CFTR functional expression. Sci Transl Med. 2014;6(246):246ra97246 ra97.

57. Cholon DM, Quinney NL, Fulcher ML et al. Potentiator ivacaftor abrogates pharmacological correction of F508 CFTR in cystic fibrosis. Sci Transl Med. 2014;6(246):246ra96-246ra96.

58. Taylor-Cousar JL, Mall MA, Ramsey BW et al. Clinical development of triplecombination CFTR modulators for cystic fibrosis patients with one or two F508del alleles. ERJ Open Res. 2019;5(2):00082-2019.

*59. Davies JC, Moskowitz SM, Brown C et al. VX-659-Tezacaftor-Ivacaftor in Patients with Cystic Fibrosis and One or Two Phe508del Alleles. N Engl J Med. 2018;379(17):1599611.

*Triple therapy VX-659-tez-iva phase 2 trial data.

*60. Keating D, Marigowda G, Burr L et al. VX-445-Tezacaftor-Ivacaftor in Patients with Cystic Fibrosis and One or Two Phe508del Alleles. N Engl J Med. 2018;379(17):1612- 
20.

*Triple therapy VX-445-tez-iva phase 2 trial data.

61. Hubert D, Dehillotte $C$, Munck A et al. Retrospective observational study of French patients with cystic fibrosis and a Gly551Asp-CFTR mutation after 1 and 2years of treatment with ivacaftor in a real-world setting. J Cyst Fibros. 2018;17(1):89-95.

62. Siracusa CM, Ryan J, Burns L et al. Electronic monitoring reveals highly variable adherence patterns in patients prescribed ivacaftor. J Cyst Fibros. 2015;14(5):621-6.

63. Trimble AT, Donaldson SH. Ivacaftor withdrawal syndrome in cystic fibrosis patients with the G551D mutation. J Cyst Fibros. 2018;17(2):e13-6.

64. Gifford A, Pearson K, Aliaj E et al. P279 Withdrawing existing chronic therapies in people with cystic fibrosis who benefit from highly effective CFTR modulator drugs. J Cyst Fibros. 2019;18:S136.

65. Roesch EA, Nichols DP, Chmiel JF. Inflammation in cystic fibrosis: An update. Pediatr Pulmonol. 2018;53(S3):S30-50.

66. Sun $X, Y i$ Y, Yan Z et al. In utero and postnatal VX-770 administration rescues multiorgan disease in a ferret model of cystic fibrosis. Sci Transl Med. 2019;11(485):eaau7531.

67. Chouchane I, Stremler-Lebel N, Reix P. Lumacaftor/ivacaftor initiation in two liver transplantation patients under tacrolimus and antifungal azoles. Clin Case Reports. $2019 ; 7(4): 616-8$.

68. Mitchell RM, Jones AM, Barry PJ. CFTR modulator therapy in patients with cystic fibrosis and an organ transplant. Paediatr Respir Rev. 2018;27:6-8.

69. Vertex Selects Triple Combination Regimen of VX-445, Tezacaftor and Ivacaftor to Submit for Global Regulatory Approvals in Cystic Fibrosis | Vertex Pharmaceuticals 
[Internet]. https://investors.vrtx.com/news-releases/news-release-details/vertexselects-triple-combination-regimen-vx-445-tezacaftor-and. 2019 [cited 2019 Jul 26].

70. Crawford KJ, Downey DG. Theratyping in cystic fibrosis. Curr Opin Pulm Med. $2018 ; 24(6): 612-7$

71. Dekkers JF, van der Ent CK, Beekman JM. Novel opportunities for CFTR-targeting drug development using organoids. Rare Dis. 2013;1(1):e27112.

72. Noordhoek J, Gulmans V, Van Der Ent K et al. Intestinal organoids and personalized medicine in cystic fibrosis: A successful patient-oriented research collaboration. Current Opinion in Pulmonary Medicine. 2016;22(6):610-6(7). 


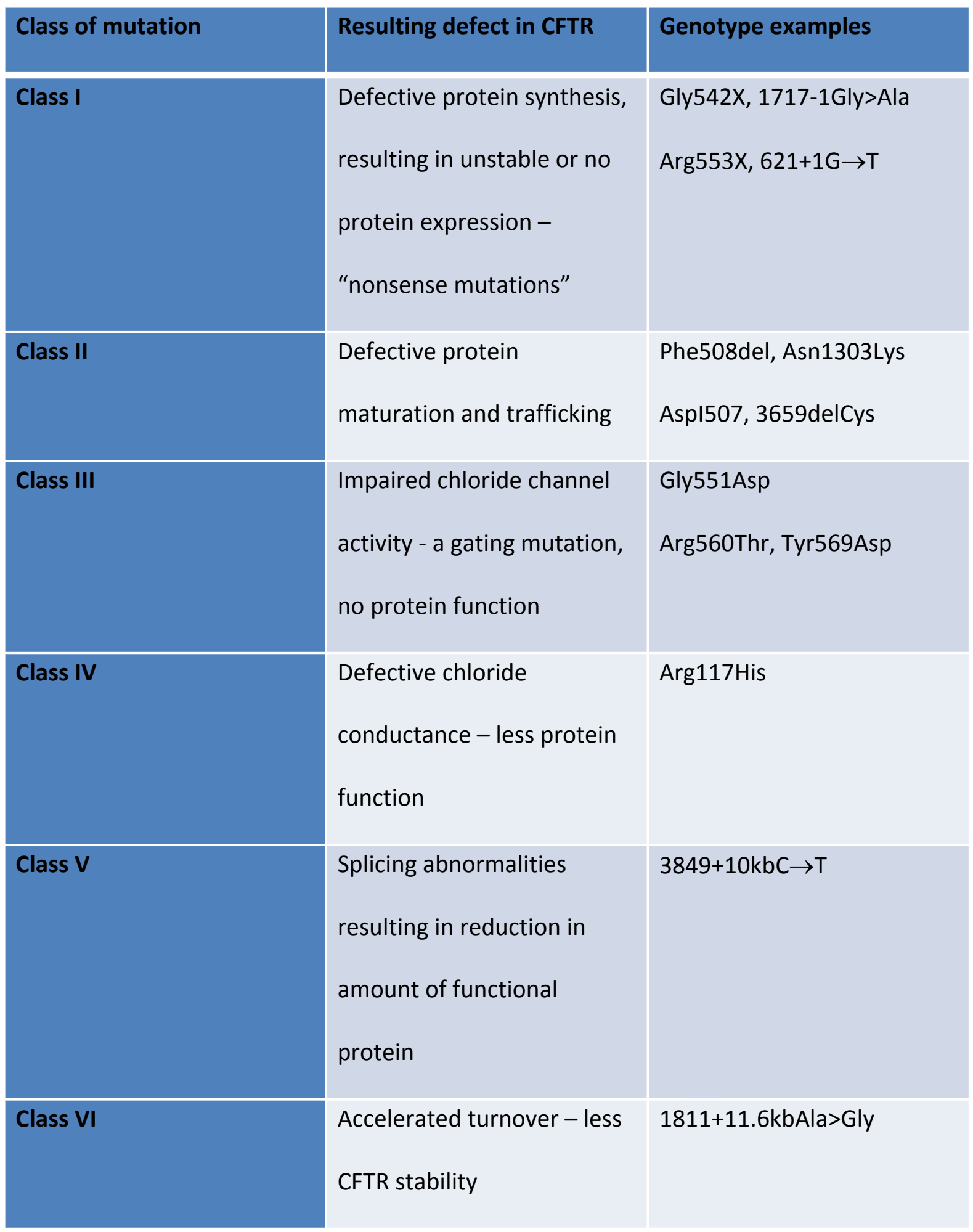

Table 1: CFTR mutation classes(7) 
Table 2: Summary of outcomes in salient CFTR modulator trials to date

1

\begin{tabular}{|c|c|c|c|c|c|c|c|c|c|c|}
\hline $\begin{array}{l}3 \\
4 \\
5 \\
6 \\
7\end{array}$ & Method & CFTR Mutation & $\begin{array}{l}\text { Inclusion } \\
\text { FEV }_{1}(\%)\end{array}$ & $\begin{array}{c}\text { Participant } \\
\text { number }\end{array}$ & $\begin{array}{c}\text { Duration } \\
\text { (weeks) }\end{array}$ & $\begin{array}{c}\Delta \text { Sweat chloride } \\
\text { (mmol/l) at week } \\
24\end{array}$ & $\begin{array}{c}\text { Absolute } \\
\Delta \text { ppFEV }_{1} \% \\
\text { (Cl) at week } 24\end{array}$ & $\begin{array}{c}\Delta \mathrm{BMI} \\
\text { improvement } \\
\mathrm{kg} / \mathrm{m}^{2} \\
\text { (CI) at week } 24\end{array}$ & $\begin{array}{c}\text { PEx rate } \\
\text { reduction } \\
\% \\
\text { (CI) at } \\
\text { week } 24\end{array}$ & $\begin{array}{c}\Delta \text { CFQ-R } \\
\text { score } \\
\text { (>4 is sig) } \\
\text { at week } 24\end{array}$ \\
\hline $\begin{array}{lc}8 & \text { STRIVE (14) } \\
9 & \\
10 & \text { Ivacaftor } \\
11 & \text { vs } \\
12 & \text { Placebo } \\
13 & \\
14 & \\
15 & 2009-2012\end{array}$ & $\begin{array}{c}\text { Phase III, } \\
\text { randomised, } \\
\text { double-blind, } \\
\text { placebo- } \\
\text { controlled. } \\
\text { Aged } \geq 12 \text { years }\end{array}$ & At least one G551Asp & $40-90$ & 161 & 48 & $\begin{array}{c}-47.9 \\
(-51.3 \text { to }-44.5)\end{array}$ & $\begin{array}{c}+10.6 \\
(8.6-12.6)\end{array}$ & $\begin{array}{c}2.8 \mathrm{~kg} \\
(1.8-3.7)\end{array}$ & $\begin{array}{c}60 \\
(36-78)\end{array}$ & $\begin{array}{c}+8.1(4.7- \\
11.4)\end{array}$ \\
\hline $\begin{array}{cc}16 & \text { ENVISION (15) } \\
17 & \\
18 & \text { Ivacaftor } \\
19 & \text { vs } \\
20 & \text { Placebo } \\
21 & \\
22 & 2009-2012\end{array}$ & $\begin{array}{c}\text { Phase III, } \\
\text { randomised, } \\
\text { double-blind, } \\
\text { placebo- } \\
\text { controlled. } \\
\text { Aged 6-11 years }\end{array}$ & At least one G551Asp & $40-105$ & 52 & 48 & -55.5 & $\begin{array}{c}+12.6 \\
(4.2-24.6)\end{array}$ & 3.7 & NM & +6.3 \\
\hline $\begin{array}{lc}23 & \text { KIWI (19) } \\
24 & \\
25 & \text { Ivacaftor } \\
26 & \\
\text { fweight adjusted dosing) } \\
27 & 50 \mathrm{mg}-\text { body weight } \\
28 & <14 \mathrm{~kg} \\
29 & 75 \mathrm{mg}-\text { body weight } \\
30 & >14 \mathrm{~kg} \\
31 & \\
32 & 2013\end{array}$ & $\begin{array}{c}\text { Open label, single } \\
\text { arm study (PK and } \\
\text { safety study) } \\
\text { Aged 2-5 years }\end{array}$ & At least one G551Asp & NA & $\begin{array}{l}\text { Part A - } 9 \\
\text { Part B - } 34\end{array}$ & 24 & -46.9 & NM & NM & NM & NM \\
\hline
\end{tabular}

33 
Table 2: Summary of outcomes in salient CFTR modulator trials to date

\begin{tabular}{|c|c|c|c|c|c|c|c|c|c|c|}
\hline $\begin{array}{lc}3 & \text { TRAFFIC/TRANSPORT } \\
4 & (31) \\
5 & \\
6 & \text { Lumacaftor/ivacaftor } \\
7 & \text { vs } \\
8 & \text { Placebo } \\
9 & \\
10 & 2013-14 \\
1\end{array}$ & $\begin{array}{c}\text { Phase III, } \\
\text { randomised, } \\
\text { double-blind, } \\
\text { placebo- } \\
\text { controlled. } \\
\text { Aged } \geq 12 \text { years }\end{array}$ & $\begin{array}{c}\text { Phe508del } \\
\text { homozygotes }\end{array}$ & $40-90$ & 954 & 24 & NM & $\begin{array}{c}+2.8 \\
(1.8-3.8)\end{array}$ & $\begin{array}{c}0.24 \\
(0.11-0.37)\end{array}$ & $\begin{array}{c}39 \\
(24-51)\end{array}$ & $\begin{array}{c}+2.2 \\
(-0.01-4.45) \\
\text { NS }\end{array}$ \\
\hline $\begin{array}{lc}12 & \\
13 & \text { NCT01531673 (43) } \\
14 & \\
15 & \\
16 & \text { vs } \\
17 & \text { Placebo } \\
18 & \\
19 & 2012-14 \\
20 & \\
21 & \\
\end{array}$ & $\begin{array}{c}\text { Phase II, } \\
\text { randomised, } \\
\text { double-blind, } \\
\text { placebo- } \\
\text { controlled. } \\
\text { Aged } \geq 18 \text { years } \\
\text { (homozygotes) } \\
\text { Aged } \geq 12 \text { years } \\
\text { (heterozygotes) }\end{array}$ & $\begin{array}{c}\text { Phe508del } \\
\text { homozygotes } \\
\text { Phe508del/G551Asp }\end{array}$ & $40-90$ & 190 & 8 & $\begin{array}{l}\text { Homozygotes: } \\
\qquad-6.04^{*} \\
\text { Heterozygotes: } \\
\text {-7.02* }\end{array}$ & $\begin{array}{l}\text { Homozygotes: } \\
+3.75^{*}(0.94- \\
6.83) \\
\text { Heterozygotes: } \\
+4.6^{*}\end{array}$ & NM & NM & $\begin{array}{l}\text { Hom: } \\
+7.62 \\
\text { Het: } \\
+3.79\end{array}$ \\
\hline $\begin{array}{lc}22 & \text { EVOLVE (44) } \\
23 & \\
24 & \\
25 & \text { vs } \\
26 & \text { Placebo } \\
27 & \\
28 & 2015-17\end{array}$ & $\begin{array}{l}\text { Phase III, } \\
\text { randomised, } \\
\text { double-blind, } \\
\text { placebo } \\
\text { controlled. } \\
\text { Aged } \geq 12 \text { years }\end{array}$ & $\begin{array}{c}\text { Phe508del } \\
\text { homozygotes }\end{array}$ & $40-90$ & 509 & 24 & $\begin{array}{c}-10.1 \\
(-11.4 \text { to }-8.8)\end{array}$ & $\begin{array}{c}+4.0 \\
(3.1-4.8)\end{array}$ & $\begin{array}{c}0.18 \\
(0.08-0.28) \\
\text { (NS }\end{array}$ & $\begin{array}{c}35 \\
(12-52) \\
\\
(0.64 \text { vs } \\
0.99 \\
\text { PEx/yr) }\end{array}$ & $\begin{array}{c}+5.1 \\
(3.2-7)\end{array}$ \\
\hline $\begin{array}{lc}29 & \text { EXPAND (45) } \\
30 & \\
31 & \text { Tezacaftor/ivacaftor } \\
32 & \text { vs } \\
33 & \text { Ivacaftor } \\
34 & \text { vs } \\
35 & \text { Placebo } \\
36 & \\
37 & 2015-17 \\
38 & \\
39 & \\
40 & \end{array}$ & $\begin{array}{c}\text { Phase III, } \\
\text { randomised, } \\
\text { double-blind } \\
\text { crossover trial. } \\
\text { Aged } \geq 12 \text { years }\end{array}$ & $\begin{array}{c}\text { Phe508del/RF } \\
\text { Eligible RF mutations: } \\
\text { 2789_5G } \rightarrow \text { A, } \\
\text { 3849+10kbC } \rightarrow \text { T, 3272- } \\
\text { 26A } \rightarrow \text { G, 711+3A } \rightarrow \text { G, } \\
\text { E56K, P67L, R74W, } \\
\text { D110ER, D110H, R117C, } \\
\text { E831X, L206W, R347H, } \\
\text { E193K, R352Q, A455E, }\end{array}$ & $40-90$ & 248 & 24 & $\begin{array}{c}-9.5^{*} \\
(-11.7 \text { to }-7.3) \\
\{-4.5(-6.7 \text { to }-2.3) \\
\text { ivacaftor group }\}\end{array}$ & $\begin{array}{c}+6.8^{*} \\
(5.7-7.8) \\
\{+4.7(3.7-5.8) \\
\text { ivacaftor group }\}\end{array}$ & $\begin{array}{c}0.34 * \text { NS } \\
\text { (0.47 ivacaftor } \\
\text { group) NS }\end{array}$ & $\begin{array}{l}29 * \text { NS } \\
\text { (0.34 vs } \\
0.63 \text { PEx } \\
\text { per yr) }\end{array}$ & $\begin{array}{c}+11.1^{*} \\
(8.6-13.7) \\
\\
\{+9.7(7.2- \\
12.2) \\
\text { ivacaftor } \\
\text { group }\}\end{array}$ \\
\hline
\end{tabular}




\section{Table 2: Summary of outcomes in salient CFTR modulator trials to date}

\begin{tabular}{|c|c|c|c|c|c|c|c|c|c|c|}
\hline $\begin{array}{l}3 \\
4 \\
5 \\
6\end{array}$ & & $\begin{array}{c}\text { D579G, S945L, S977F, } \\
\text { F1052V, K1060T, } \\
\text { R1070W, F1074L, } \\
\text { D1152H, D1270N }\end{array}$ & & & & & & & & \\
\hline $\begin{array}{|lc|}7 & \text { NCT02953314 (48) } \\
8 & \text { Tezacaftor/ivacaftor } \\
9 & \\
10 & 2018 \\
11 & \\
12 & \\
13 & \\
14 & \\
\end{array}$ & $\begin{array}{l}\text { Phase III, open } \\
\text { label, safety and } \\
\text { tolerability trial. } \\
\text { Aged 6-11 years }\end{array}$ & $\begin{array}{c}\text { Phe508del } \\
\text { homozygotes }\end{array}$ & $\geq 40$ & $\begin{array}{l}\text { Part A - } 13 \\
\text { Part B - } 70\end{array}$ & 24 & $\begin{array}{c}-14.5 \\
(-17.4 \text { to }-11.6)\end{array}$ & $\begin{array}{c}+1.4 \\
(-0.4-3.1)\end{array}$ & $\begin{array}{c}0.23 \\
(0.06-0.4)\end{array}$ & NM & NM \\
\hline 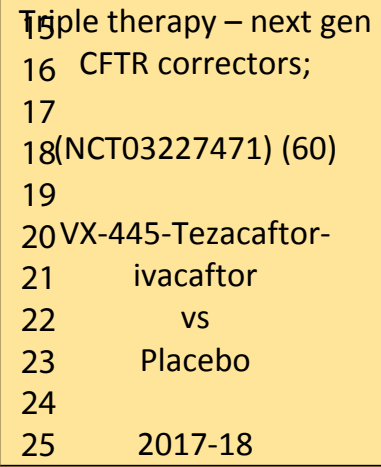 & $\begin{array}{l}\text { Phase II, } \\
\text { randomised, } \\
\text { double-blind, } \\
\text { placebo-or active } \\
\text { controlled, } \\
\text { parallel-group } \\
\geq 18 \text { years }\end{array}$ & $\begin{array}{c}\text { Phe508del } \\
\text { homozygotes } \\
\text { Phe508del/MF }\end{array}$ & $40-90$ & 123 & $\begin{array}{l}4 \text { (29 } \\
\text { days) }\end{array}$ & $\begin{array}{c}\text { Phe508del/MF: } \\
-39.1^{* *} \\
\text { (-44.9 to }-33.3) \\
\text { Phe508del hom: } \\
-39.6^{* *} \\
\text { (-45.3 to }-33.8)\end{array}$ & $\begin{array}{c}\text { Phe508del + MF: } \\
+13.8^{* *} \\
(10.9-16.6) \\
\text { Phe508del hom: } \\
+11.0^{* *} \\
(7.9-14.0)\end{array}$ & NM & NM & $\begin{array}{c}\text { Phe508del/ } \\
\text { MF: } \\
+25.7^{* *} \\
(18.3-33.1) \\
\text { Phe508del } \\
\text { Hom: } \\
+20.7^{* *} \\
(12.5-29)\end{array}$ \\
\hline 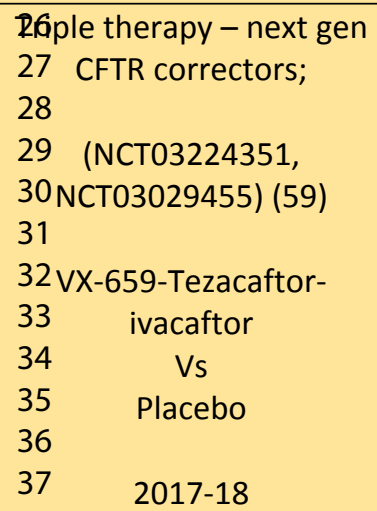 & $\begin{array}{l}\text { Phase II, } \\
\text { randomised, } \\
\text { double-blind, } \\
\text { placebo-or or } \\
\text { active-controlled, } \\
\text { parallel group } \\
\geq 18 \text { years }\end{array}$ & $\begin{array}{c}\text { Phe508del } \\
\text { homozygotes } \\
\text { Phe508del/MF }\end{array}$ & $40-90$ & 117 & $\begin{array}{l}4 \text { (29 } \\
\text { days) }\end{array}$ & $\begin{array}{c}\text { Phe508del/MF: } \\
-51.4^{* *} \\
\text { (-57.8 to }-44.9) \\
\text { Phe508del hom: } \\
-42.4^{* *} \\
\text { (-46.8 to }-37.7)\end{array}$ & $\begin{array}{c}\text { Phe508del/MF: } \\
+13.3^{* *} \\
(9.5-17.1) \\
\\
\text { Phe508del hom: } \\
+9.7^{* *} \\
(6.6-12.7)\end{array}$ & NM & NM & $\begin{array}{c}\text { Phe508del/ } \\
\text { MF: } \\
+21.8^{* *} \\
(13.6-30.0) \\
\\
\\
\text { Phe508del } \\
\text { Hom: } \\
+19.5^{* *} \\
(13.1-25.9)\end{array}$ \\
\hline
\end{tabular}

$\mathrm{ppFEV}_{1}$ - percentage of predicted forced expiratory volume in one second, $\mathrm{Cl}$ - confidence interval, $\mathrm{PEx}$ - pulmonary exacerbation, $\mathrm{BMI}-$ body mass index, $\mathrm{CFQ}-\mathrm{R}$ - cystic fibrosis

questionnaire - respiratory domain, hom - homozygous, RF - residual function, MF - minimal function. *Results at 8 weeks, ${ }^{* *}$ Results at day 29 , NM - not measured, NS - not significant 
Table 2: Summary of outcomes in salient CFTR modulator trials to date 
Table 3: Pharmacology, drug-drug interactions, monitoring and dosing recommendations of CFTR modulators

\begin{tabular}{|c|c|c|c|c|c|c|c|}
\hline $\begin{array}{c}\text { CFTR } \\
\text { modulator }\end{array}$ & $\begin{array}{l}\text { Mechanism } \\
\text { of action }\end{array}$ & $\begin{array}{l}\text { Pharmacokinetics } \\
\text { (PK) }\end{array}$ & $\begin{array}{l}\text { Mechanism of } \\
\text { DDls }\end{array}$ & $\begin{array}{c}\text { Monitoring and } \\
\text { special } \\
\text { considerations }\end{array}$ & $\begin{array}{l}\text { Contraindications } \\
\text { (Cl) }\end{array}$ & Dosing & $\begin{array}{c}\text { Clinical } \\
\text { indication }\end{array}$ \\
\hline $\begin{array}{l}\text { Ivacaftor(1) } \\
\text { VX-770 } \\
\text { Kalydeco }^{\circledR}\end{array}$ & $\begin{array}{c}\text { CFTR } \\
\text { potentiator } \\
\text { Improves CFTR } \\
\text { anion channel } \\
\text { open probability } \\
\left(P_{0}\right) \text { at cell } \\
\text { surface }\end{array}$ & $\begin{array}{l}\text { Small molecule } \\
\text { Metabolised in liver } \\
\text { (CYP3A4) into } \\
\text { metabolites M1 and } \\
\text { M6 } \\
\text { Excreted in } \\
\text { bile/faeces, negligible } \\
\text { in urine } \\
\text { Half-life 12-14 hours } \\
\text { Higher absorption } \\
\text { with fat-containing } \\
\text { foods - increases AUC } \\
\text { x2.5-4 } \\
\text { CYP3A4 substrate and } \\
\text { weak inhibitor }\end{array}$ & $\begin{array}{c}\text { CYP3A4 inhibitors } \\
\text { (increase ivacaftor } \\
\text { exposure): Azole } \\
\text { antifungals, macrolides } \\
\text { (not including } \\
\text { azithromycin), } \\
\text { immunosuppressants, } \\
\text { benzodiazepines. } \\
\text { CYP34A inducers } \\
\text { (decrease ivacaftor } \\
\text { exposure): } \\
\text { rifampicin/rifabutin }\end{array}$ & $\begin{array}{l}\text { Liver function monitoring: } \\
\text { Baseline, } 3 \text { monthly for } \\
\text { one year then annually } \\
\text { Requires dose reduction } \\
\text { in CP* class B/C liver } \\
\text { insufficiency } \\
\text { Decrease ivacaftor dose } \\
\text { with co-administration of } \\
\text { azole and macrolides } \\
\text { Monitor } \\
\text { immunosuppressant } \\
\text { levels, may need } \\
\text { decreased dose with } \\
\text { ivacaftor } \\
\text { Avoid combination of } \\
\text { rifampicin/rifabutin with } \\
\text { ivacaftor }\end{array}$ & $\begin{array}{l}\text { Absolute: } \\
\text { Hypersensitivity } \\
\text { Relative: } \\
\text { 1. Severe liver } \\
\text { impairment } \\
\text { (CP class C) } \\
\text { Caution in } \\
\text { eGFR } \\
<30 \mathrm{ml} / \mathrm{min} / 1 . \\
73 \mathrm{~m}^{2} \text { and } \\
\text { ESRD }\end{array}$ & $\begin{array}{l}\text { 150mg } \\
\text { twice daily } \\
\text { (if }>25 \mathrm{~kg} \text { ) }\end{array}$ & $\begin{array}{c}\text { Monotherapy: } \\
\text { Aged } \geq 6 \text { years: } \\
\text { Class III CFTR gating } \\
\text { mutations: } \\
\text { Gly551Asp } \\
\text { Gly178Arg } \\
\text { Ser549Arg } \\
\text { Ser549Asn } \\
\text { Gly551Ser } \\
\text { Gly970Arg } \\
\text { Gly1244Glu } \\
\text { Ser1251Asn } \\
\text { Ser1255Pro } \\
\text { Gly1349Asp } \\
\text { (+further } 23 \text { RF } \\
\text { mutations in } \\
\text { patients } \geq 2 \text { years in } \\
\text { US) } \\
\text { And, } \\
\text { Aged } \geq 18 \text { years: } \\
\text { Class IV - Arg117His }\end{array}$ \\
\hline
\end{tabular}




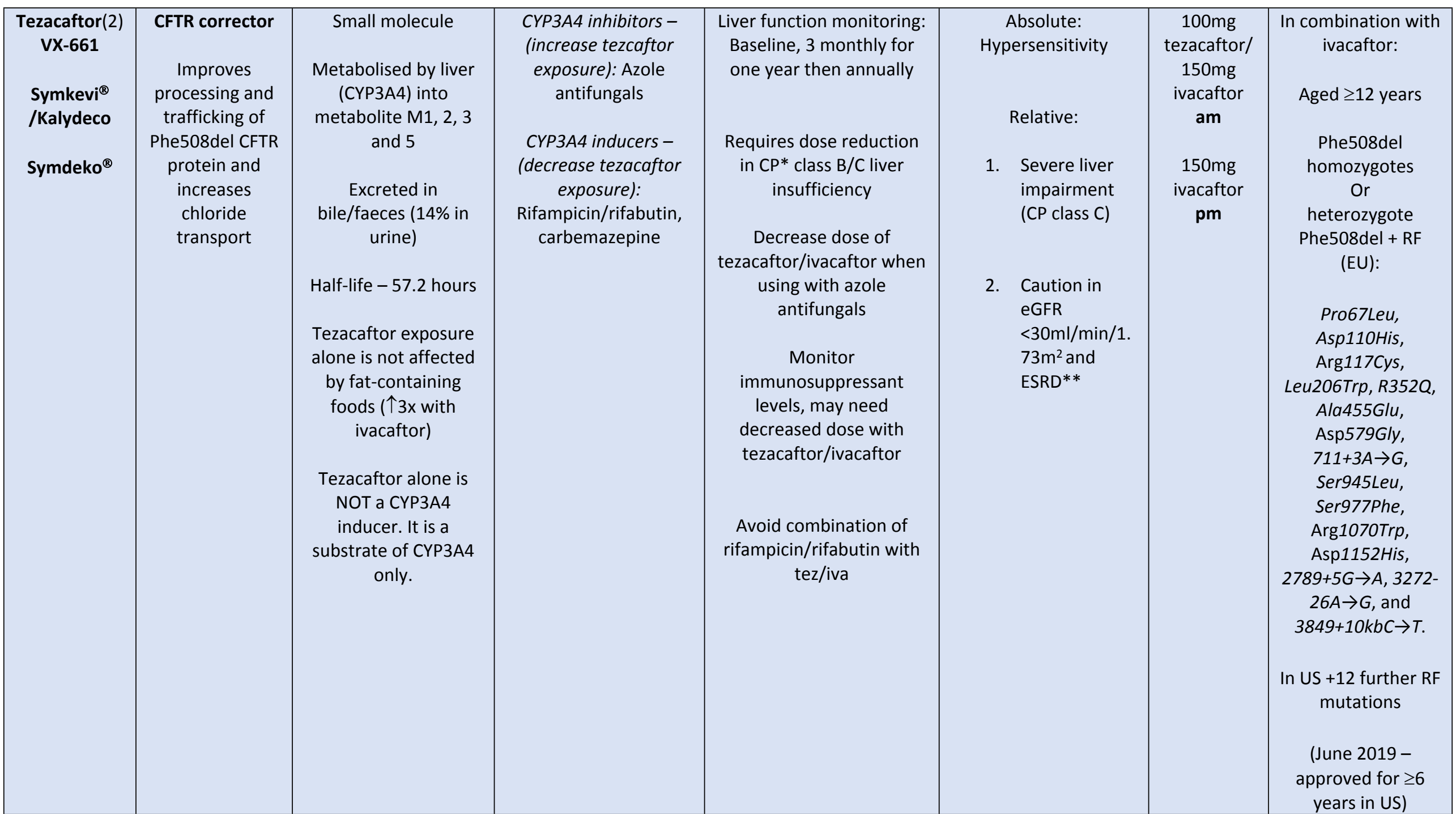

1. Kalydeco $150 \mathrm{mg}$ film-coated tablets - Summary of Product Characteristics (SmPC) - (eMC) [Internet]. EMC. 2017 [cited 2019 Jul 26 ]. Available from: https://www.medicines.org.uk/emc/product/3040/smpc.

2. Symkevi $100 \mathrm{mg} / 150 \mathrm{mg}$ film coated tablets - Summary of Product Characteristics (SmPC) - (eMC) [Internet]. EMC. 2018 [cited 2019 Jul 26]. Available from: 
https://www.medicines.org.uk/emc/product/9634/smpc.

CFTR - cystic fibrosis transmembrane conductance regulator, AUC - area under the curve, eGFR-estimated glomerular filtration rate, RF - residual function, EU - European Union, US - United States.

*Child-Pugh liver score (CP)

**End-stage renal disease (ESRD) - eGFR $<30 \mathrm{ml} / \mathrm{min}$ 


\begin{tabular}{|c|c|}
\hline In vivo efficacy & In vitro efficacy \\
\hline homozygote & $\begin{array}{l}\text { 711+3A } \rightarrow \text { G, 2789-5G } \rightarrow \text { A, 3272-26A } \rightarrow \mathrm{G}, \\
\text { 3846_10kbC } \rightarrow \text { T, Ala455Glu, Ala1067Thr, } \\
\text { Asp110Glu, Asp110His, Asp579Gly, } \\
\text { Asp1152His, Asp1270Asn, Glu56Lys, } \\
\text { Glu193lys, Glu831X, Phe1052Val, } \\
\text { Phe1074Leu, Lys1060Thr, Leu206Trp, } \\
\text { Pro67Leu, Arg74Trp, Arg117Cys, Arg347His, } \\
\text { Arg352Gln, Arg1070Trp, Ser945Leu, } \\
\text { Ser977Phe }\end{array}$ \\
\hline
\end{tabular}

Table 4: In vitro and in vivo efficacy of tezacaftor/ivacaftor for different CFTR mutations. 


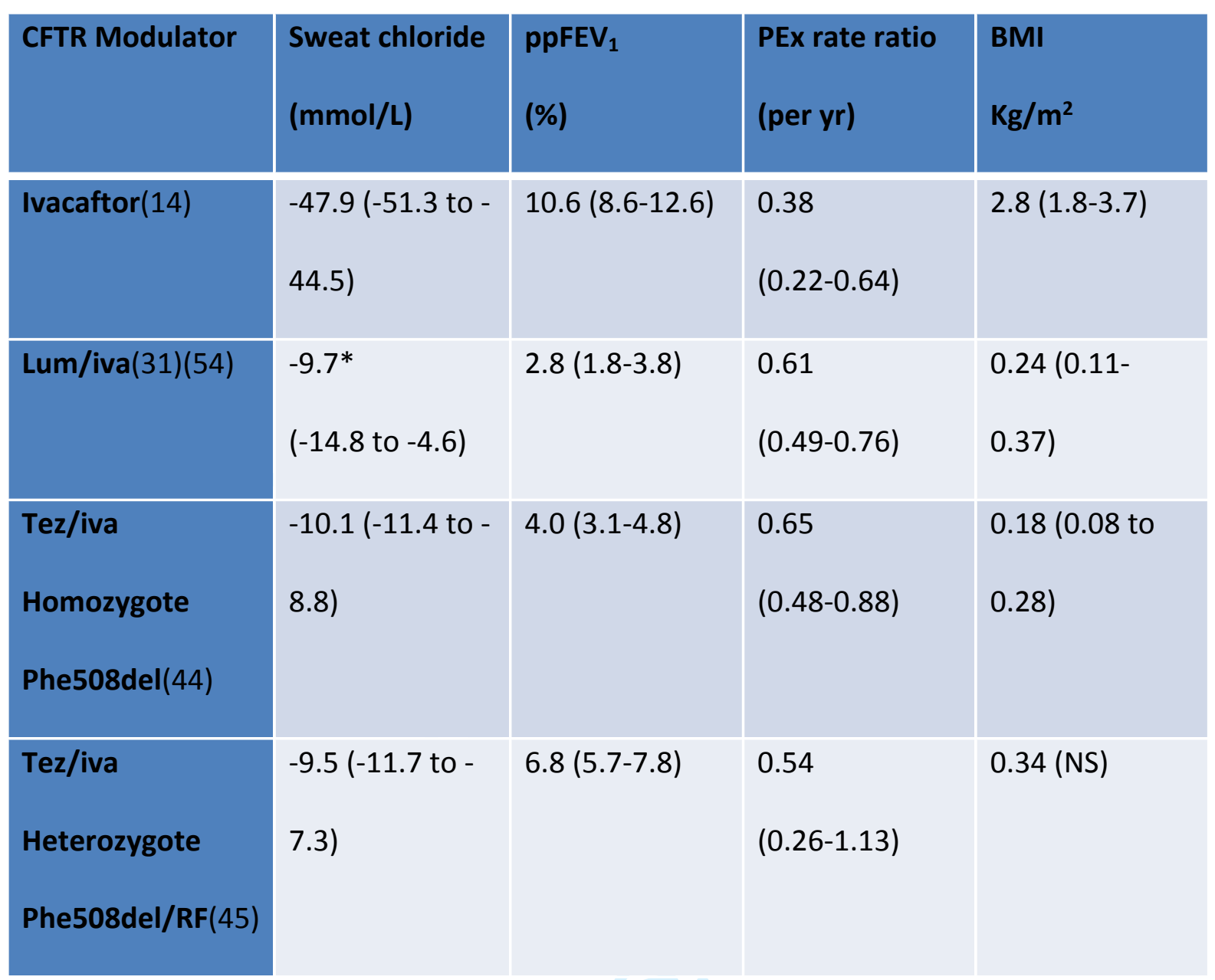

Table 5: Clinical efficacy of CFTR modulators from phase 3 trials in patients aged 12 years and over, unless otherwise stated.

* Phase 2 trial lumacaftor/ivacaftor data 


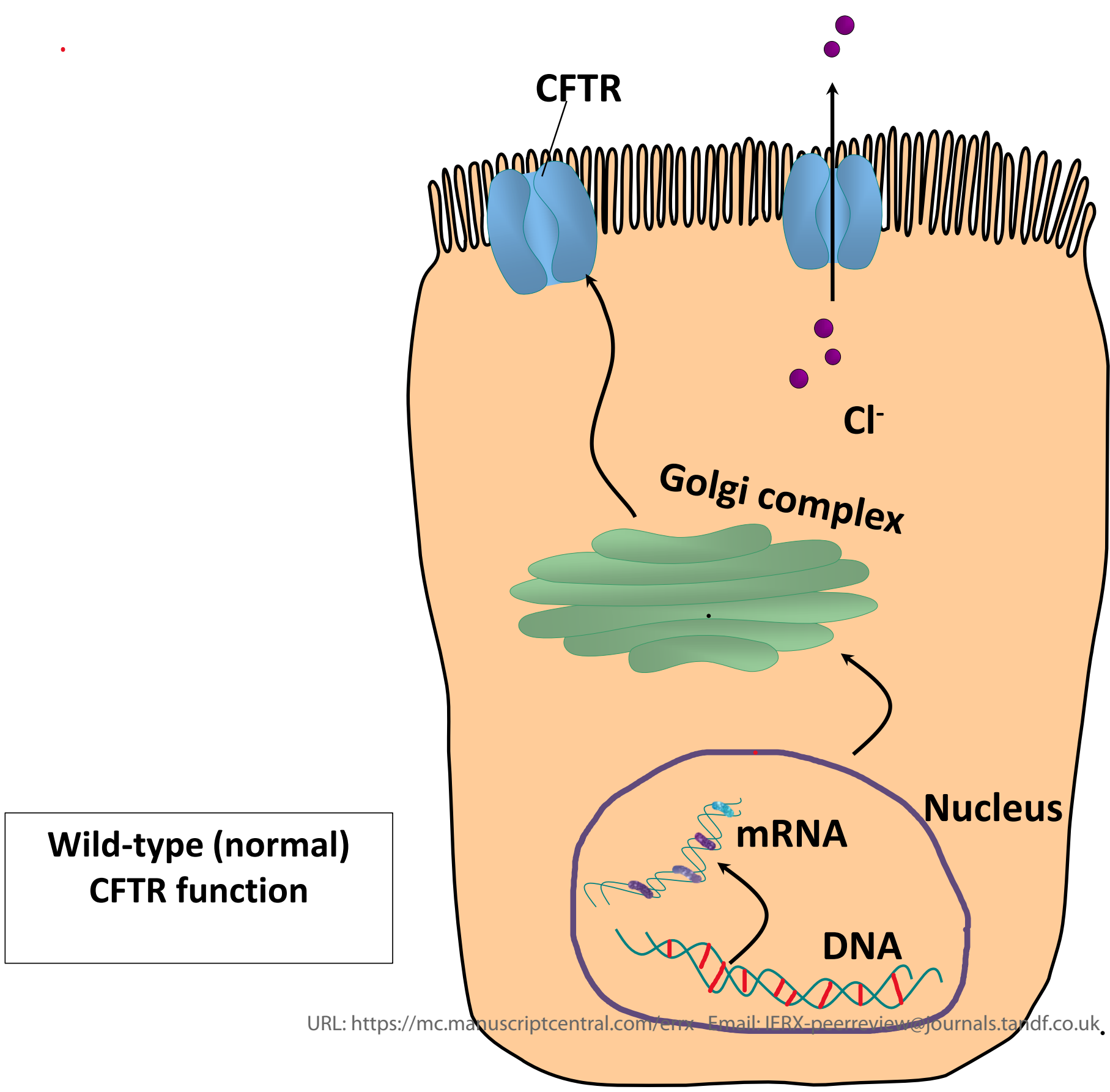




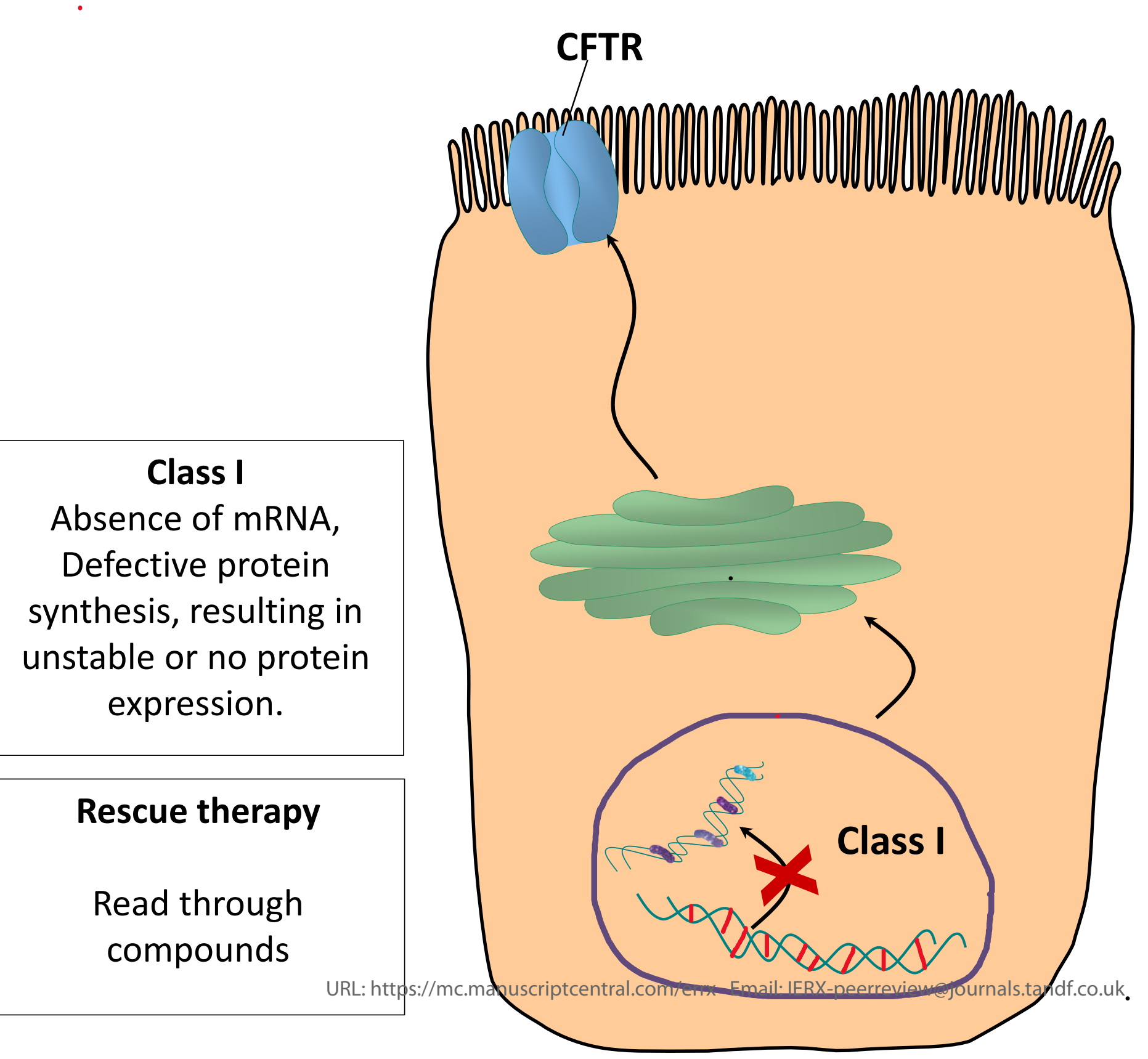




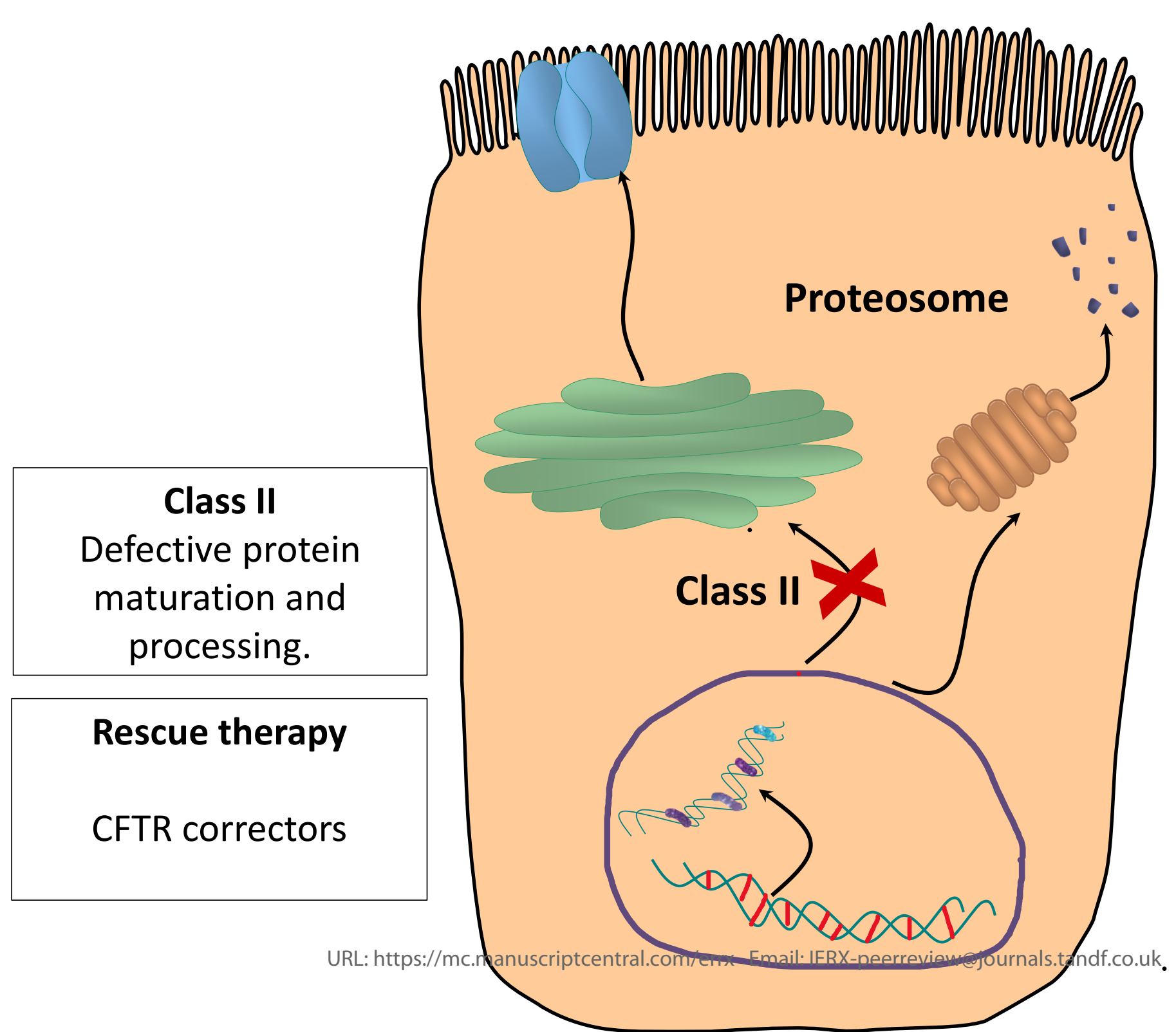




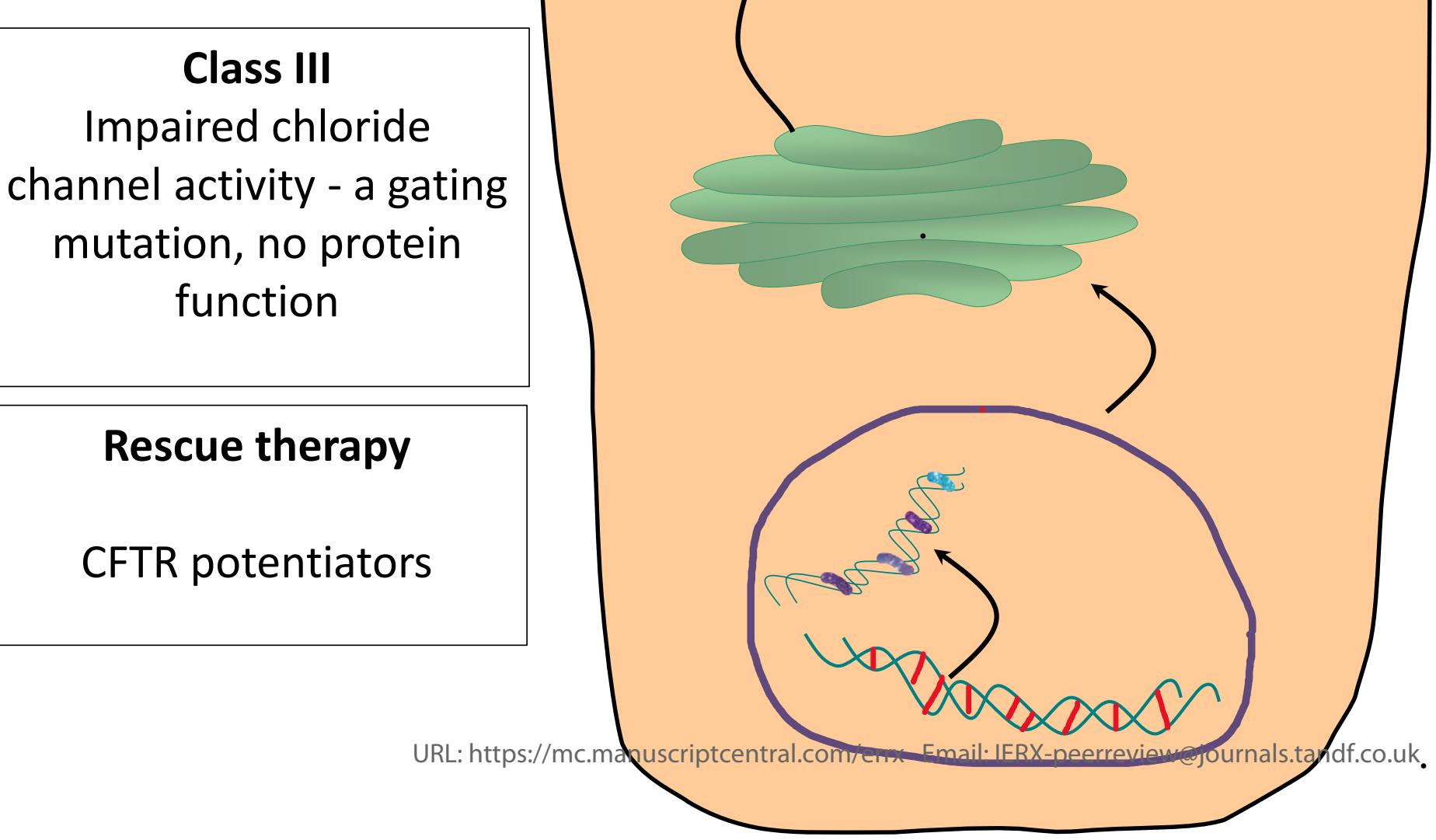




\section{Class IV}

Defective chloride conductance, less protein function.

\section{Rescue therapy}

CFTR potentiators

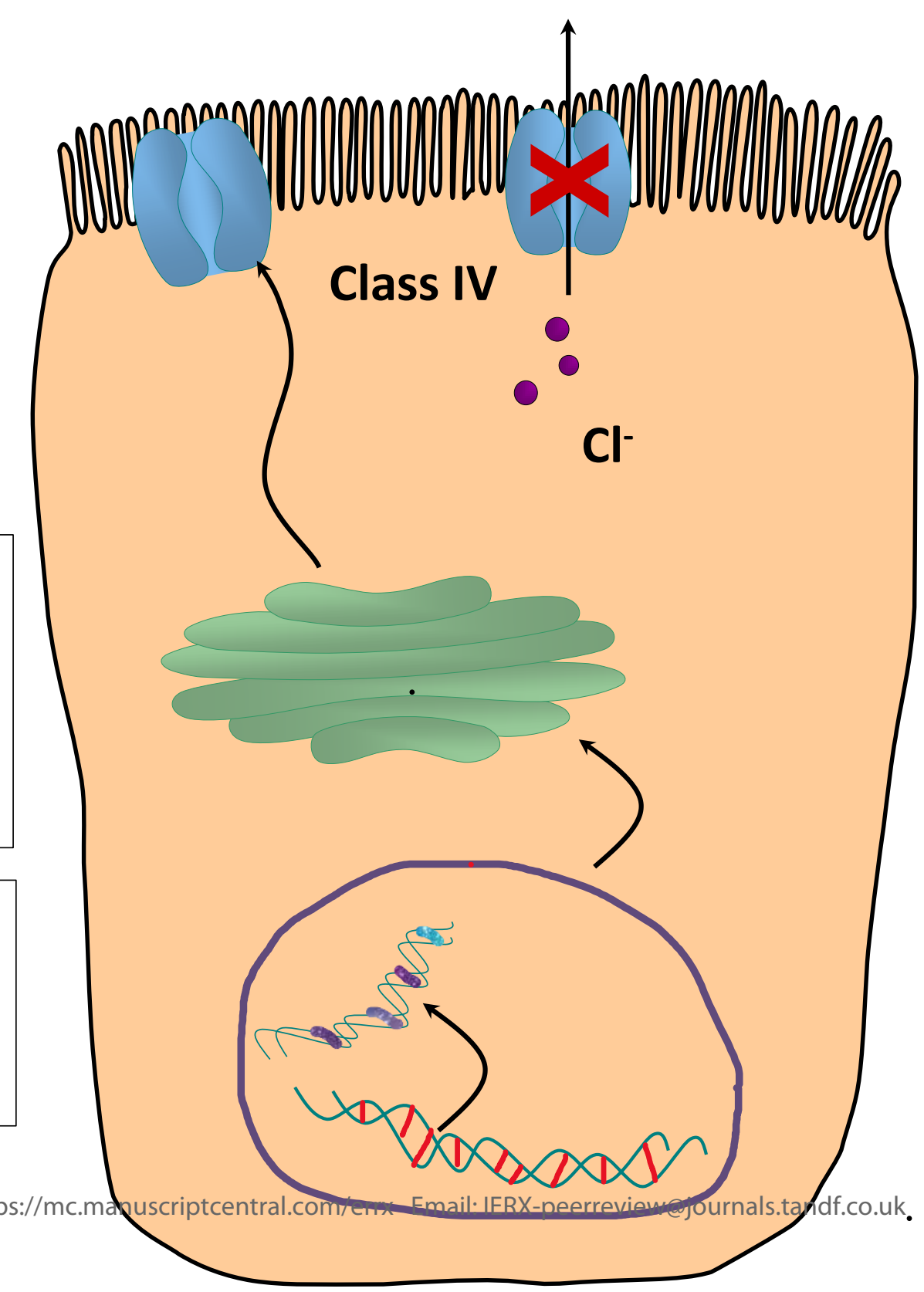




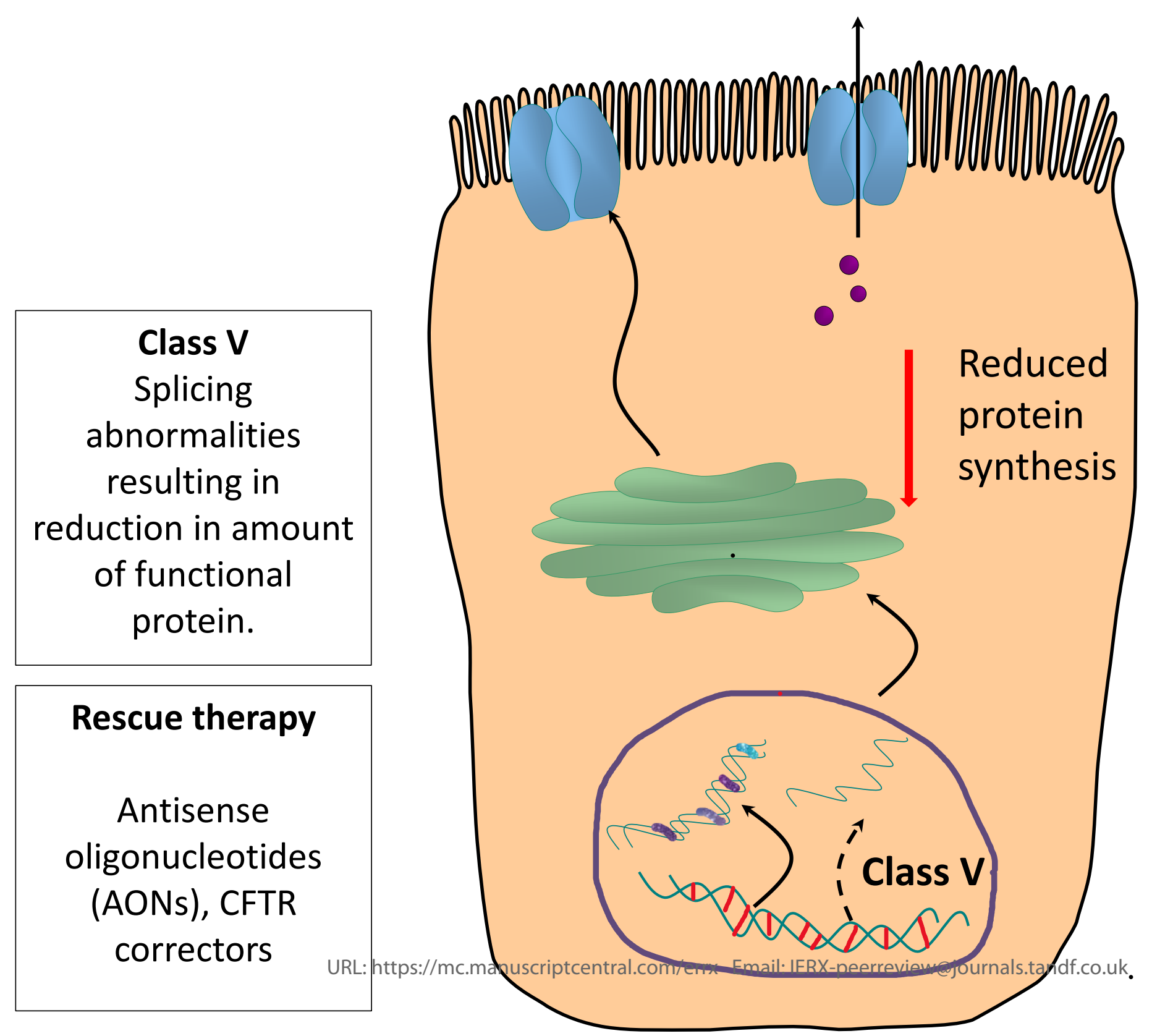




\begin{tabular}{|c|}
\hline Class VI \\
Accelerated \\
turnover - less \\
CFTR stability.
\end{tabular}

\section{Rescue therapy}

CFTR stabilisers

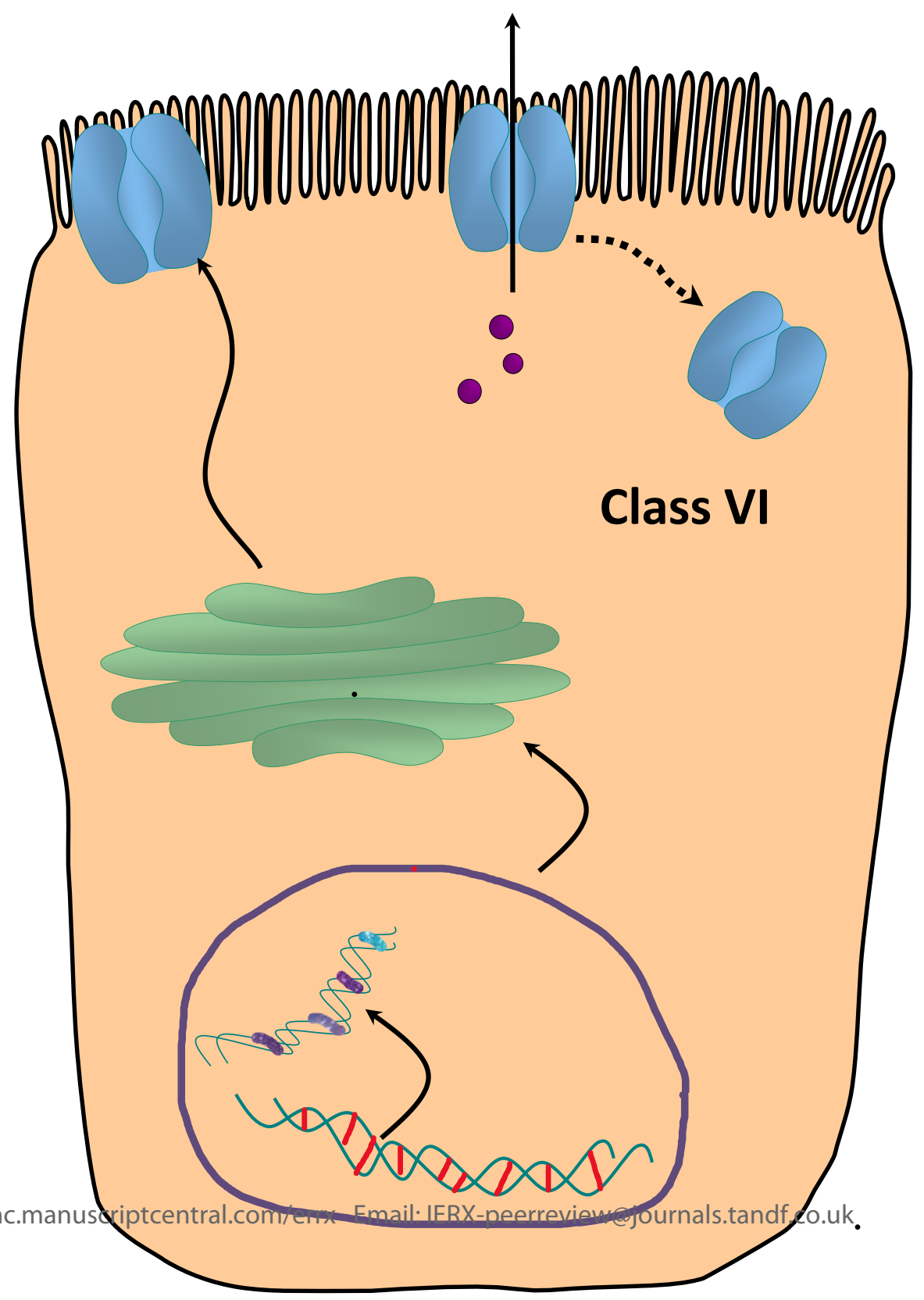




\section{Figure 2aChange in baseline sweat chloride - CFTR modulators vs placebo.}

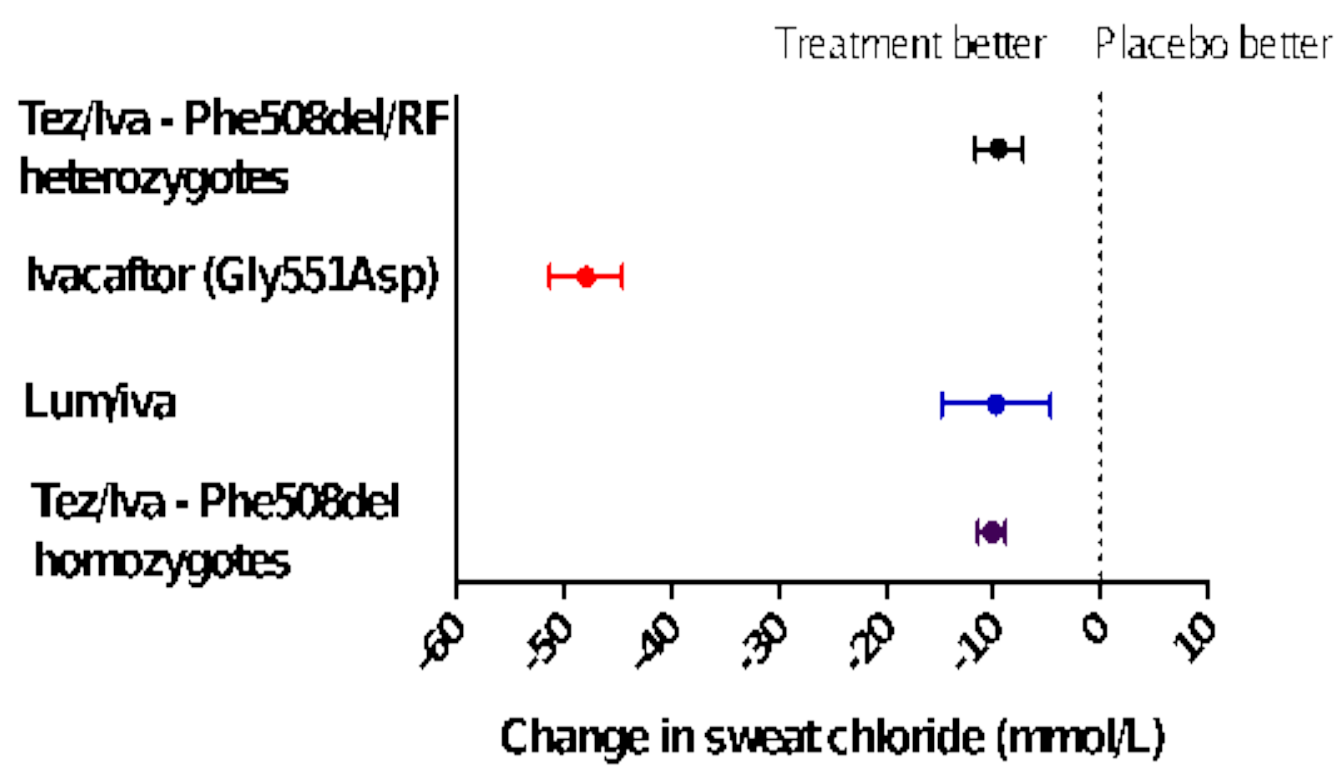




\section{Figure 2bChange in absolute ppFEVCFTR modulators vs placebo.}

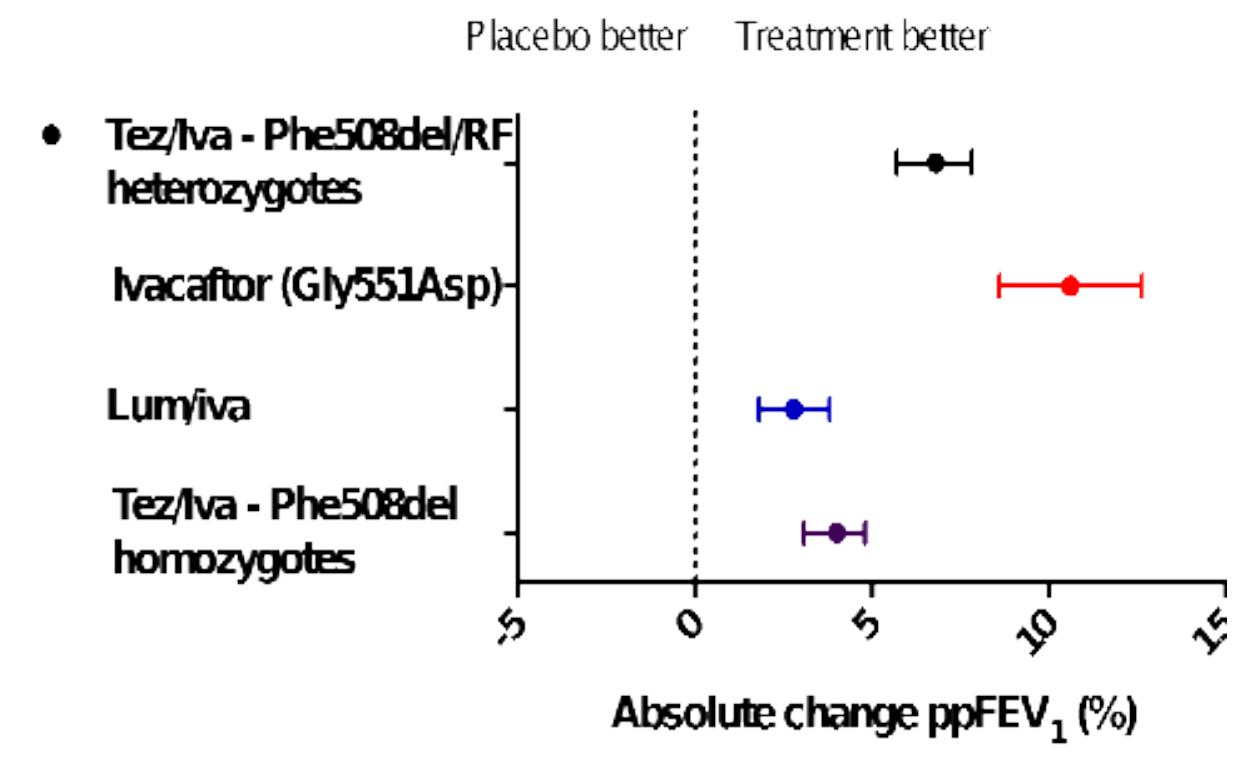




\section{Figure 2cComparison of pulmonary exacerbation risk ratio - CFTR modulators vs placebo.}

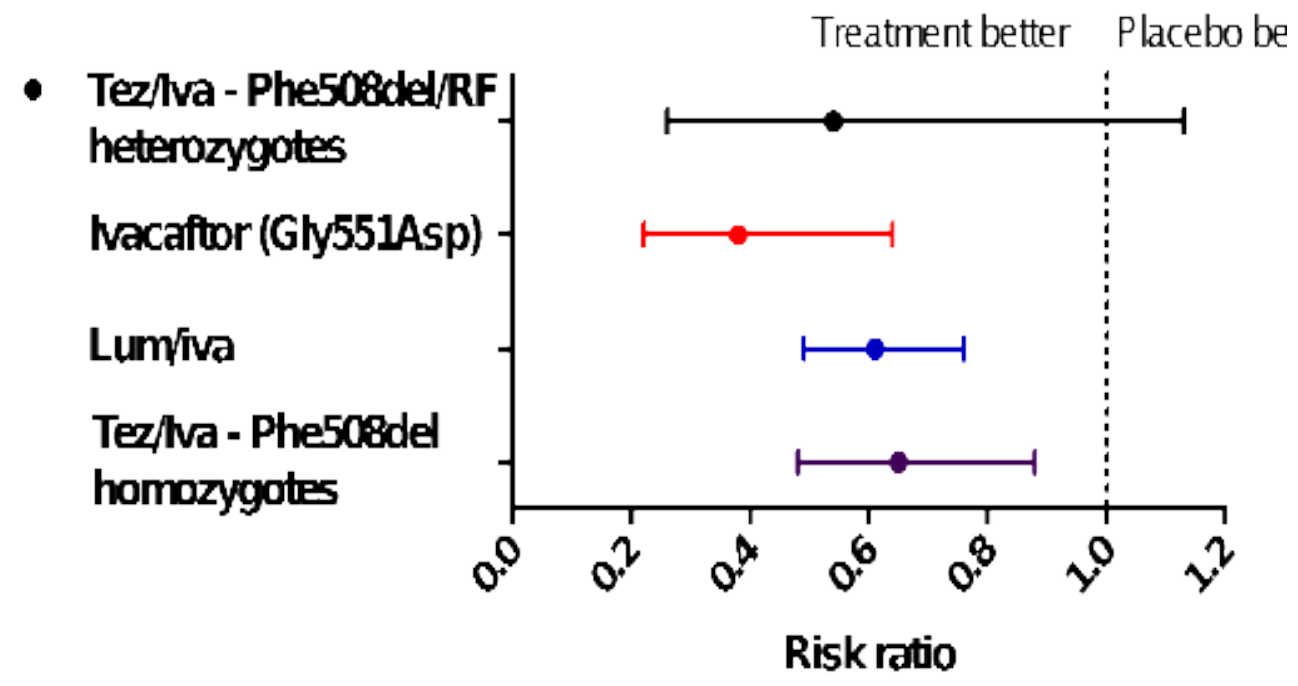




\section{Full legend for figure:}

Figure 2Graphical comparisons of trial primary outcome measures between currently available CFTR modulators. $R F$ - residual function, ppFEVpercentage of predicted forced expiratory volume in one secortdx/iva tezacaftor/ivacaftor, lum/iva - lumacaftor/ivacaftor.

2a: Change in baseline sweat chloride - CFTR modulators vs placebo.

2b: Change in absolute ppFEVCFTR modulators vs placebo.

2c: Comparison of pulmonary exacerbation risk ratio - CFTR modulators vs placebo. 